\title{
13. UPPER CENOZOIC SILICOFLAGELLATES FROM OFFSHORE ECUADOR, DEEP SEA DRILLING PROJECT SITE 5041
}

\author{
David Bukry, United States Geological Survey, Scripps Institution of Oceanography, La Jolla, California
}

\begin{abstract}
Diverse and abundant late Miocene to Pleistocene silicoflagellates at DSDP Site 504 can be correlated by tropical biostratigraphic zones and relative paleotemperature values to eastern tropical Pacific reference site DSDP 503A farther to the west. Early Pliocene assemblages, which were poorly known until now, are present and can be correlated locally between DSDP Holes 504, 503A, and 495, using species events associated with the new Dictyocha pulchella Subzone and Dictyocha angulata Subzone. Silicoflagellate relative paleotemperature values show major warming at 4.7 to $5.0 \mathrm{Ma}$ (Cores 45-48), 3.4 to 3.8 Ma (Cores 32-33), 1.5 to 1.7 Ma (Cores 12-16), and 0.5 to 0.8 Ma (Cores 3-6). Major coolings occurred at 5.0 to $5.1 \mathrm{Ma}$ (Core 51 ), 3.9 to $4.4 \mathrm{Ma}$ (Cores 38-44), and 1.0 to $1.3 \mathrm{Ma}$ (Cores 8-10). The appearance of Dictyocha longa is proposed to replace the asperoid/fibuloid ratio reversal as the bottom of the Dictyocha fibula Zone, because the non-evolutionary ratio reverses several times in the upper Miocene of Hole 503A, and at least once in Hole 504. Three new Pliocene silicoflagellates are defined: Dictyocha concinna Bukry, n. sp., D. helix Bukry, $\mathrm{n}$. sp., and D. tamarae Bukry, n. sp.
\end{abstract}

\section{INTRODUCTION}

A 237- to 264-meter section of rapidly accumulated pelagic sediment blankets the igneous basement in the DSDP Site 504 area of the Costa Rica Rift $\left(1^{\circ} 13.58 \mathrm{~N}\right.$, $83^{\circ} 43.93 \mathrm{~W}$; depth $3460 \mathrm{~m}$ ). Hole 504 is an excellent reference section for latest Miocene to Pleistocene silicoflagellates (Figs. 1A-C). The 54 hydraulic-piston cores (HPC) cut in Hole 504 at this site provide a detailed, undisturbed sequence of biostratigraphic and paleoecologic events. This site was chosen to study geothermal problems in a young, non-rifted, flat area of oceanic crust that is cooling by simple conduction.

The low-latitude biostratigraphic zonation of silicoflagellates is recognized throughout the section by primary guide species. Redefinition of the base of the Dictyocha fibula Zone in the upper Miocene was required by the reversal or high readings for the asperoid/fibuloid ratio in upper Miocene Cores 49 to 53. Previous problems with the biostratigraphic use of this ratio were described for DSDP Hole 503A (Bukry, in press a). Several variants of distorted Mesocena quadrangula from the same upper Miocene interval may have local biostratigraphic significance because they are missing in younger populations (Bukry and Foster, 1973); these variants are illustrated (Plate 9).

Calculations of paleotemperature values $\left(T_{\mathrm{s}}\right)$ from quantitative silicoflagellate data and the application of diatom chronology for DSDP Hole 504 show cooling at approximately 5.0 to $5.1 \mathrm{Ma}, 3.9$ to $4.4 \mathrm{Ma}$, and 1.0 to $1.3 \mathrm{Ma}$, and major warming at 4.7 to $5.0 \mathrm{Ma}, 3.4$ to 3.8 $\mathrm{Ma}$, and 1.5 to $1.7 \mathrm{Ma}$. Some of these peaks are correlated between sites and discussed because they are associated with several species events at both DSDP Hole 503A and DSDP Hole 504. A revision to the $T_{\mathrm{s}}$ equation

\footnotetext{
${ }^{1}$ Cann, J. R., Langseth, M. G., Honnorez, J., Von Herzen, R. P., White, S. M., et al., Init. Repts. DSDP, 69: Washington (U.S. Govt. Printing Office).
}

is made to accommodate large abundances $(73 \%)$ of Mesocena quadrangula in the Pleistocene.

\section{METHODS AND MATERIALS}

As for previous eastern tropical Pacific (ETP) photomicroscope silicoflagellate studies of DSDP Legs $16,34,54,63,67$, and 68 , sediment samples were cleaned in 250 -ml beakers with $\mathrm{H}_{2} \mathrm{O}_{2}(35 \%), \mathrm{HCl}$ (conc.) and $\mathrm{H}_{2} \mathrm{O}$ (distilled), using low heat from a hot plate to speed chemical digestion of carbonate, organic matter, and clay from the biosiliceous residue. After settlings and rinsings in $\mathrm{H}_{2} \mathrm{O}$ (distilled), the residue was bottled. Slides were prepared by re-suspending the whole acid residue by shaking the storage bottle and quickly pipetting 2 or 3 drops onto a glass slide. A metal spatula was used to spread the suspension evenly over the slide. This was dried at medium heat on a hot plate and sealed with a coverslip, using a thermoplastic mounting medium (Piccolyte). Mechanical stage traverses of all, or representative, slide areas and a mechanical counter were used to enumerate all the silicoflagellates encountered at magnifications of $200 \times$ to $500 \times$, most commonly $250 \times$, until 300 specimens were counted.

\section{NEOGENE ZONATION}

Similar ranges of several new species of silicoflagellates at DSDP Sites 504, 503, and 495 justify identification of two new subzones for the lower Pliocene and upper Miocene. A preliminary chronology is assigned to selected silicoflagellate events that are most compatible with the diatom chronology (see Sancetta, this volume). The zonation is defined in Bukry (in press a, b) and briefly characterized below, relative to the assemblages from DSDP Site 504 from youngest to oldest.

\section{Dictyocha aculeata Zone}

The Dictyocha aculeata Zone is identified by the common occurrence of Dictyocha aculeata above the Quaternary acme of Mesocena quadrangula. At DSDP Hole 504 , Core 6 contains a transitional assemblage with 3 to $9 \% D$. aculeata and $4 \% M$. quadrangula. Because $M$. quadrangula is missing in shallower cores, this Core 6 assemblage is assigned to the upper $M$. quadrangula Zone. According to Burckle (1977), the extinction of $M$. quadrangula occurs above the Jaramillo magnetic event 
(approximately $0.79 \mathrm{Ma}$ ). At DSDP Sites 504 and 503, and some Leg 54 sites, the $M$. quadrangula extinction occurred near that of the diatom Nitzschia reinholdii, at approximately $0.63 \mathrm{Ma}$ (see Barron, 1980). In fact, for Hole 503A, shipboard results showed the $M$. quadrangula extinction to be slightly younger than the $N$. reinholdii in Core 4.

\section{Mesocena quadrangula Zone}

The Mesocena quadrangula Zone at Site 504 includes the Quaternary acme of Mesocena quadrangula (abundances up to $73 \%$ ) in Cores 6 to 11 . Although M. quadrangula is common (11-14\%) in lower Quaternary Cores 12 and 13, the presence of Dictyocha delicata identifies the upper $D$. delicata Subzone of the underlying Dictyocha stapedia Zone in the eastern tropical Pacific. Auxiliary stratigraphic guides from higher-latitude areas, such as Dictyocha hessii, D. lingii, or $D$. subarctios, are missing. Among the low-latitude species present, D. perlaevis perlaevis displaces $M$. quadrangula as a dominant species near the top of the zone, which suggests that a warming trend contributed to the final disappearance of M. quadrangula.

\section{Dictyocha stapedia Zone}

\section{Dictyocha delicata Subzone}

The regional extinction of $D$. delicata is used to mark the top of the Dictyocha delicata Subzone. The base is the first common occurrence of $D$. delicata, which includes the uppermost Pliocene and lower Quaternary below the Mesocena quadrangula Zone. At Site 504, Cores 12 to 18 are assigned to the $D$. delicata Subzone; however, the nominate species is missing in samples from Cores 16 and 17. Instead, a similar taxon, D. tamarae, is present there. The highest significant populations $(>5 \%)$ of Distephanus for the subzone occur up through the middle of the subzone. The Quaternary species Octactis pulchra appears only at the top of the zone, after Distephanus has declined.

\section{Dictyocha ornata Subzone}

The Dictyocha ornata Subzone at DSDP Site 504 has common Dictyocha ornata africana in the lower part and sparse $D$. ornata ornata in the upper part. As at Atlantic sites and other eastern tropical Pacific (ETP) sites, the range and common occurrence of $D$. perlaevis flexatella is similar to $D$. ornata. Both taxa are most abundant in Cores 24 to 27, in the lower upper Pliocene. Other typical events for this subzone in the ETP that are duplicated at Site 504 are the extinction of $D$. longa, the first abundant $D$. stapedia stapedia, and the final decline or extinction of $D$. brevispina. A new correlation horizon within the $D$. ornata Subzone is suggested by the distribution of $D$. concinna. This species occurs in sediments representing a brief time and is present in moderate numbers $(5 \%$ and $7 \%)$ at the same stratigraphic position at DSDP Holes 503A and 504. The conjunction of these occurrences and the age-depth plot for diatom index species at these sites yields an estimated age of $2.3 \mathrm{Ma}$ for the $D$. concinna horizon. This is also the level of the extinction of $D$. longa at the two sites. The first $D$. ornata and $D$. perlaevis flexatella occur in Core 27 , which according to shipboard diatom chronology lies between the first Rhizosolenia praebergonii (2.92 $\mathrm{Ma})$ and the last Nitzschia jouseae (2.48 Ma).

\section{Dictyocha fibula Zone, Redefined}

The lower part of Site 504, in Cores 30 to 53, is assigned to the uppermost Miocene and lower Pliocene part of the Dictyocha fibula Zone on the basis of the low asperoid/fibuloid ratio, the occurrence of Dictyocha longa throughout the zone, and the sparse occurrence of species appearing in the Miocene $D$. neonautica Subzone of Cores 52 or 53. Asperoid taxa dominate Dictyocha in Cores 51 and 53 and are numerically important in Cores 48 to 50, and 52. (See Dictyocha brevispina Zone redefinition, below). Fairly long-ranged species such as Distephanus speculum and Dictyocha perlaevis are common; however, the disappearance of common Dictyocha pulchella and the appearance of $D$. angulata are closely spaced in the lower Pliocene (Cores 42-47) and help to divide the zone. This same sequence is also suggested in the lower Pliocene at Site 503 between Cores 21 and 27, and at Site 495 between Cores 11 and 13.

The base of the Dictyocha fibula Zone is redefined herein to be the first common occurrence of Dictyocha longa. Although assemblages above this level generally have asperoid/fibuloid ratios of less than one, the ratios are auxiliary criteria and not a prerequisite for identifying the redefined Dictyocha fibula Zone.

\section{Dictyocha angulata Subzone}

The new Dictyocha angulata Subzone is defined herein as the interval from the first occurrence to the last common Dictyocha angulata, which occupies the upper part of the Dictyocha fibula Zone, but may not extend completely to the top of the zone. The subzone is limited to the Pliocene in the known occurrences in the ETP at DSDP 495 (Core 11), 503A (Cores 20 and 21), and 504 (Cores 36-42) (see Bukry, in press a, c, for assemblages).

\section{Dictyocha pulchella Subzone}

The new Dictyocha pulchella Subzone is defined herein as the interval from the last Dictyocha neonautica to the last common $D$. pulchella. Long-ranging $D$. pulchella is common and consistently present into the lower Pliocene, permitting identification of the subzone at DSDP Hole 504 (Cores 47-53), DSDP Hole 503A (Cores 27-32), and DSDP Hole 495 (Core 13). These DSDP sites and holes corner a large triangular area in the ETP; however, at northwestern Pacific DSDP Site 310 the subzone is not recognized (Bukry, 1978a) and thus may be only of regional significance.

\section{Dictyocha neonautica Subzone}

The upper Miocene Dictyocha neonautica Subzone is not fully developed at DSDP Site 504 . Only sparse specimens characteristic of the subzone occur in the basal Core 54. The specimens of $D$. neonautica var. cocosen- 
sis, $D$. sp. aff. $D$. subclinata, and $D$. transenna are sparse, but probably represent the top of the $D$. neonautica Subzone.

\section{Dictyocha brevispina Zone, Redefined}

The original upper boundary of this zone is the change from minor-axis bar domination (asperoid) in Dictyocha to major-axis bar domination (fibuloid). It is now recognized that this shift is not permanent, but has reversed several times at various sites (Bukry, in press a, b). The shift in bar orientation is independent of various taxonomic preferences because Martini's (1971) systematics were broadly based. The asperoid/fibuloid ratio shift of dominance between species of Dictyocha is only a general lower boundary guide because it can reverse and create difficulty in zonal nomination.

A possible evolutionary replacement criterion was suggested for the ETP by the first common occurrence of Dictyocha longa, which appeared (7.1-7.2 Ma, according to Leg 68 diatom datums) near the time of the first(?) asperoid/fibuloid shift in the late Miocene (Bukry, in press a). This event occurs just below the base of the lower Dictyocha neonautica Subzone, and therefore the redefinition of the zone boundary should not require any reassignment of subzonal biostratigraphic units. Although $D$. longa is designated as the zone boundary species, the appearance of $D$. neonautica is only slightly higher at DSDP 503A (7.0-7.1 Ma, according to diatom datums), and could serve as an auxiliary marker species in the ETP.

\section{PALEOTEMPERATURE VALUES}

Paleotemperature values $\left(T_{\mathrm{s}}\right)$ calculated from the abundances of warm and temperate silicoflagellate genera (Bukry, in press d) appear in the species distribution figures (Fig. 1A-C). Several major paleotemperature trends which correlate with similar trends identified by a combination of diatom and silicoflagellate species events at DSDP Hole 503A occur where comparable HPC stratigraphic control is available (Figs. 2 and 3). Rotary coring and the broader sample interval at DSDP Hole 495 in the northern ETP prohibit such detailed comparison. The paleotemperature record for DSDP Hole 504 covers a partially younger interval $(0.5-5.2 \mathrm{Ma})$ than DSDP Hole 503A (2.5-7.4 Ma); therefore, the overlap between the two sites extends from 2.5 to $5.2 \mathrm{Ma}$.

Mesocena quadrangula Peak (late Miocene, 5.1 Ma)

DSDP Holes $503 \mathrm{~A}$ and 504 both show a local maximum in the abundance of $M$. quadrangula $(21 \%$ and $19 \%$ ) at about 5.1 Ma, in the latest Miocene, in a fibuloid-dominated assemblage. The paleotemperature values for these coeval occurrences range from $T_{\mathrm{s}} 50$ to 80 at DSDP Hole 503A and $T_{\mathrm{s}} 50$ to 74 at DSDP 504. Further sampling of this event is needed.

\section{Mesocena quadrangula/Dictyocha pulchella Warm Peak (early Pliocene, $\sim 5.0 \mathrm{Ma}$ )}

Following the period of oscillating lower paleotemperature values in the latest Miocene of DSDP Hole
$503 \mathrm{~A}$, a warm peak at about 4.9 to $5.0 \mathrm{Ma}$ is indicated by $T_{\mathrm{s}}$ values up to 92 at DSDP Hole $503 \mathrm{~A}$ and 85 at DSDP Hole 504. A local reduction in M. quadrangula and Distephanus speculum s. ampl. at this peak indicates warming prior to the disappearance of $D$. pulchella.

Dictyocha pulchella/Dictyocha angulata Cool Peak (early Pliocene, 3.9-4.4 Ma)

Following the disappearance of Dictyocha pulchella, a moderate but fairly broad cool peak, culminating at about 4.1 Ma, occurs at both DSDP Holes 503A and 504. The $T_{\mathrm{s}}$ values for this peak at DSDP Hole 504 are considerably cooler (42-61) than at DSDP Hole 503A (68-72), and sample coverage at DSDP Hole 504 reveals a warming to $T_{\mathrm{s}} 88$ which is superimposed on the broader cooling trend. This superimposed and perhaps local warm peak $\left(T_{s} 88\right)$ at DSDP Hole 504 occurs just before 4.2 $\mathrm{Ma}$ and interrupts the broader trend for a decline from $T_{\mathrm{s}} 61$ to $T_{\mathrm{s}} 42$. The $D$. pulchella/D. angulata cool peak between about 3.9 to $4.4 \mathrm{Ma}$ correlates with the early Pliocene glacial event between 4.2 and 4.3 Ma suggested by Keigwin (1979) at DSDP Site 158.

\section{Dictyocha angulata Warm Peak ( 3.4-3.8 Ma)}

A distinctive, broad warm peak in $T_{\mathrm{s}}$ values occurs at both DSDP Holes 503A and 504, with the same apex value of $T_{\mathrm{s}} 97$ between about 3.4 and 3.8 Ma. Dictyocha angulata disappeared during this period, a maximum $T_{\mathrm{s}}$ occurring near 3.6 Ma. Data on DSDP Hole 503A are insufficient to subdivide the chronologic record from 3.2 to $3.6 \mathrm{Ma}$; however, the 3.5-Ma Thalassiosira convexa diatom event coincides with part of a silicoflagellate warm peak, establishing a general upper limit near 3.5 Ma for this warm peak at DSDP Hole 503A.

Mesocena Cool Peak (early Quaternary, $\sim 1.0-1.3 \mathrm{Ma}$ ) and Dictyocha aculeata Warm Peak (late Quaternary, 0.8 to ? Ma)

Minor $T_{\mathrm{s}}$ oscillations through the late Pliocene at DSDP Hole 504 cannot be correlated, because of a paucity of matching data for DSDP Hole 503A. The Quaternary Mesocena acme cooling (to $T_{\mathrm{s}} 63$ from the Quaternary high of $T_{\mathrm{s}} 91$ ) and the following Dictyocha aculeata warming (to $T_{\mathrm{s}} 98$ ) are the most distinctive features of the $T_{s}$ record for the younger strata of DSDP Hole 504.

The degree of cooling suggested for the early Pleistocene from approximately 1.0 to $1.3 \mathrm{Ma}$ by the original calculation of $T_{\mathrm{s}}$ values ranging from 20 to 40 was exaggerated by the extremely prolific bloom of Mesocena quadrangula at DSDP Hole 504. Because an increase in the percentage of cold-water Distephanus (hexagonal) did not occur, a much more moderate cooling must be accepted. This is expressed by the addition of $M$. quadrangula as part of the temperate factor in the calculation of $T_{\mathrm{s}}$ values. The cooling trend during the $M$. quadrangula blooms would then be of a magnitude comparable to those of the late Pliocene. Therefore, $T_{\mathrm{s}}$ value calculations should add all quadrate Mesocena to the summation $T_{\mathrm{s}}=X_{\mathrm{w}}+0.5 X_{\mathrm{t}}$ as part of the temperate factor $X_{\mathrm{t}}$. 


\begin{tabular}{|c|c|c|c|c|c|c|c|c|c|c|c|c|c|c|c|c|c|}
\hline Age & \multicolumn{12}{|c|}{ Early Pliocene } & \multicolumn{5}{|c|}{ Late Miocene } \\
\hline Zone & \multicolumn{12}{|c|}{ D. fibula } & & & * & & $*$ \\
\hline Subzone & \multicolumn{7}{|c|}{ D. angulata } & \multicolumn{3}{|c|}{-} & \multicolumn{6}{|c|}{ D. pulchella } & D. $n$. \\
\hline Sub-bottom Depth (m) & $\stackrel{?}{5}$ & $\begin{array}{l}\infty \\
\infty \\
0 \\
0\end{array}$ & $\stackrel{\check{I}}{\Xi}$ & $\stackrel{\sim}{\infty}$ & 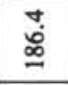 & ¿্் & $\stackrel{+}{\stackrel{\Delta}{a}}$ & ఫே & $\stackrel{\square}{\stackrel{0}{9}}$ & ડ્తి & さั & $\underset{d}{\dot{d}}$ & $\underset{\sim}{\stackrel{\Delta}{*}}$ & ส̇ & $\begin{array}{l}\text { đั. } \\
\text { สี่ }\end{array}$ & ণ্ণ & ले \\
\hline Taxa & $\begin{array}{l}\text { U } \\
\text { D. }\end{array}$ & 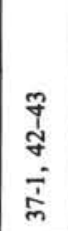 & 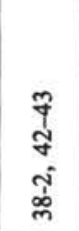 & 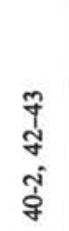 & $\begin{array}{l}\text { İ } \\
\text { q } \\
\dot{z} \\
\dot{z}\end{array}$ & 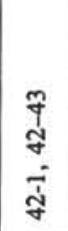 & 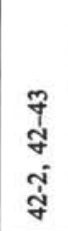 & $\begin{array}{l}\text { qै } \\
\text { f } \\
\dot{y} \\
\dot{y}\end{array}$ & 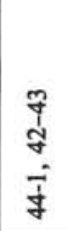 & \begin{tabular}{l} 
qे \\
\multirow{y}{*}{} \\
$\vec{j}$
\end{tabular} & 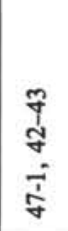 & $\begin{array}{l}\text { qे } \\
\text { q } \\
\dot{j} \\
\dot{q}\end{array}$ & 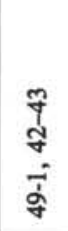 & $\begin{array}{l}\text { ปे } \\
\text { के } \\
\text { के }\end{array}$ & $\begin{array}{l}\text { ָे } \\
\text { भ } \\
\frac{\pi}{\pi}\end{array}$ & 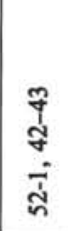 & స̃ \\
\hline $\begin{array}{l}\text { Dictyocha angulata } \\
D \text {. sp. cf. } D \text {. angulata } \\
D \text {. aspera aspera } \\
\text { D. aspera clinata s. ampl. } \\
\text { D. brevispina }\end{array}$ & 28 & 21 & $<1$ & $<1$ & 7 & 30 & $\begin{array}{r}25 \\
1 \\
4\end{array}$ & 3 & 10 & 1 & 1 & 12 & 1 & $\begin{array}{r}1 \\
<1 \\
7 \\
\end{array}$ & $\begin{array}{r}1 \\
1 \\
12 \\
\end{array}$ & 1 & 4 \\
\hline $\begin{array}{l}\text { D. brevispina (no bar) } \\
\text { D. brevispina (giant asymmetric) } \\
\text { D. concavata } \\
\text { D. fibula s. ampl. } \\
\text { D. longa }\end{array}$ & $\begin{array}{r}2 \\
14 \\
\end{array}$ & $\begin{array}{l}2 \\
7 \\
\end{array}$ & $\begin{array}{r}1 \\
3 \\
16 \\
\end{array}$ & $\begin{array}{l}5 \\
7 \\
\end{array}$ & $\begin{array}{l}6 \\
1 \\
\end{array}$ & $\begin{array}{r}5 \\
5 \\
15 \\
\end{array}$ & $\begin{array}{r}4 \\
22 \\
\end{array}$ & $\begin{array}{r}<1 \\
4 \\
20\end{array}$ & $\begin{array}{r}2 \\
12 \\
\end{array}$ & $\begin{array}{r}5 \\
28 \\
\end{array}$ & 7 & $\begin{array}{r}1 \\
12 \\
\end{array}$ & 2 & $\begin{array}{l}\mathrm{X} \\
2 \\
2 \\
\end{array}$ & $\begin{array}{l}1 \\
1 \\
\end{array}$ & $\begin{array}{l}2 \\
3 \\
\end{array}$ & $\begin{array}{l}2 \\
7 \\
3 \\
\end{array}$ \\
\hline $\begin{array}{l}\text { D. longa var. paxilla } \\
\text { D. perfecta } \\
\text { D. perlaevis } \\
D . \text { pulchella } \\
D . \text { sp. aff. } D \text {. pulchella (angular) }\end{array}$ & $\begin{array}{r}5 \\
36\end{array}$ & $\begin{array}{r}<1 \\
7 \\
27\end{array}$ & $\begin{array}{r}4 \\
2 \\
12 \\
1\end{array}$ & $\begin{array}{r}3 \\
20 \\
7 \\
3\end{array}$ & $\begin{array}{r}4 \\
11 \\
30\end{array}$ & $\begin{array}{r}18 \\
2 \\
3 \\
1\end{array}$ & $\begin{array}{l}6 \\
1\end{array}$ & $\begin{array}{r}3 \\
21 \\
<1\end{array}$ & $\begin{array}{r}5 \\
8 \\
12 \\
1\end{array}$ & $\begin{array}{r}10 \\
7 \\
8\end{array}$ & $\begin{array}{l}23 \\
18 \\
10 \\
13\end{array}$ & $\begin{array}{r}22 \\
5 \\
6 \\
22\end{array}$ & $\begin{array}{r}14 \\
1 \\
9 \\
21\end{array}$ & $\begin{array}{r}18 \\
1 \\
15\end{array}$ & $\begin{array}{r}9 \\
<1 \\
24 \\
1\end{array}$ & $\begin{array}{r}6 \\
13 \\
8 \\
14 \\
10 \\
\end{array}$ & $\begin{array}{r}2 \\
<1 \\
1 \\
18 \\
4\end{array}$ \\
\hline $\begin{array}{l}\text { D. neonautica var. cocosensis } \\
D . \text { sp. aff. } D \text {. subclinata } \\
D . \text { transenna } \\
D . \text { sp. aff. D. transenna (spined) } \\
D . \text { varia } \\
\end{array}$ & & & & & & & & 1 & & & & $\begin{array}{l}<1 \\
<1\end{array}$ & & & & $\begin{array}{r}\mathrm{X} \\
<1 \\
1 \\
\end{array}$ & $\begin{array}{r}<1 \\
2 \\
1\end{array}$ \\
\hline $\begin{array}{l}\text { D. spp. } \\
\text { Distephanus boliviensis } \\
\text { D. crux crux s. ampl. } \\
\text { D. crux bispinosus } \\
\text { D. crux carolae } \\
\end{array}$ & $\begin{array}{r}4 \\
<1 \\
<1\end{array}$ & $\begin{array}{r}5 \\
1 \\
\\
<1\end{array}$ & $\begin{array}{r}1 \\
<1 \\
1\end{array}$ & $\begin{array}{r}10 \\
1 \\
<1\end{array}$ & 9 & 3 & $\begin{array}{r}11 \\
<1 \\
1 \\
1\end{array}$ & 17 & $\begin{array}{l}5 \\
2 \\
2 \\
4\end{array}$ & $\begin{array}{r}6 \\
<1 \\
5\end{array}$ & 12 & 3 & $\begin{array}{l}3 \\
2\end{array}$ & $\begin{array}{l}2 \\
1\end{array}$ & $\begin{array}{r}<1 \\
2\end{array}$ & $\begin{array}{r}4 \\
<1 \\
\end{array}$ & 3 \\
\hline $\begin{array}{l}\text { D. sp. cf. } D \text {. frugalis } \\
D . \text { mesophthalmus } \\
\text { D. polyactis } \\
\text { D. quinquangellus } \\
\text { D. speculum bispicatus }\end{array}$ & 4 & 5 & 27 & $\begin{array}{r}<1 \\
<1 \\
5\end{array}$ & 4 & 2 & $\begin{array}{l}1 \\
9\end{array}$ & $\begin{array}{r}<1 \\
12\end{array}$ & $\begin{array}{r}<1 \\
4\end{array}$ & $\begin{array}{r}<1 \\
<1 \\
1\end{array}$ & & 3 & $\begin{array}{r}<1 \\
10\end{array}$ & 3 & $\begin{array}{r}<1 \\
4\end{array}$ & $\begin{array}{l}1 \\
3 \\
\end{array}$ & $\begin{array}{r}<1 \\
2\end{array}$ \\
\hline $\begin{array}{l}\text { D. speculum f. coronata } \\
\text { D. speculum minutus } \\
D . \text { speculum speculum } \\
\text { D. speculum tenuis } \\
D \text {. sp. A (Bukry, 1979) } \\
\end{array}$ & 3 & $\begin{array}{r}14 \\
<1\end{array}$ & $\begin{array}{r}32 \\
<1\end{array}$ & $\begin{array}{r}<1 \\
34\end{array}$ & $\begin{array}{r}20 \\
2 \\
\end{array}$ & $\begin{array}{r}7 \\
<1 \\
\end{array}$ & 12 & $\begin{array}{r}9 \\
<1\end{array}$ & 23 & 12 & 9 & 10 & 22 & 34 & 40 & 14 & 44 \\
\hline $\begin{array}{l}\text { Mesocena circulus } \\
\text { M. diodon nodosa } \\
\text { M. quadrangula } \\
\text { M. quadrangula (distorted) } \\
\text { M. quadrangula (transennoid) } \\
\end{array}$ & 1 & & $<1 R$ & 2 & 5 & 2 & 2 & $\begin{array}{l}4 \\
4\end{array}$ & $\begin{array}{l}6 \\
2\end{array}$ & $\begin{array}{r}8 \\
\times \\
\end{array}$ & & $<1$ & $<1$ & $\begin{array}{r}13 \\
<1\end{array}$ & $\begin{array}{r}<1 \\
2\end{array}$ & $\begin{array}{r}1 \\
8 \\
11\end{array}$ & $\begin{array}{r}<1 \\
\mathrm{x}^{6} \\
\end{array}$ \\
\hline M. triodon & & & & & & & & & & & & + & & & & 1 & \\
\hline Total Specimens & 300 & 300 & 300 & 300 & 300 & 300 & 300 & 300 & 300 & 300 & 100 & 300 & 300 & 300 & 300 & 300 & 300 \\
\hline Paleotemperature Value & 92 & 81 & 42 & 57 & 72 & 88 & 75 & 72 & 62 & 80 & 84 & 85 & 68 & 56 & 53 & 74 & 50 \\
\hline
\end{tabular}

Figure 1. A. Silicoflagellates from Cores 3 to 53 of DSDP Hole 504 recorded as percentages. The asperoid/fibuloid ratio is greater than one in Cores 51 and $53\left({ }^{*}\right)$, which would have indicated the Dictyocha brevispina Zone prior to a redefinition of that zone herein; D. $n$. = Dictyocha neonautica; $\mathrm{X}=$ recorded after count; $\mathrm{R}=$ probably a reworked occurrence.

\section{LOWER PLIOCENE SILICOFLAGELLATE CORRELATION FOR THE EASTERN TROPICAL PACIFIC OCEAN}

The lower Pliocene is one of the least-known intervals in silicoflagellate biostratigraphy, but recent hydrau- lic-piston coring and rotary-coring operations by $\mathrm{D} / \mathrm{V}$ Glomar Challenger in the eastern tropical Pacific Ocean have provided three excellent new reference sections at DSDP Holes $495\left(12^{\circ} 29.78 \mathrm{~N}, 91^{\circ} 02.26 \mathrm{~W}\right.$; depth 4140 $\mathrm{m}), 503 \mathrm{~A}\left(4^{\circ} 04.04 \mathrm{~N}, 95^{\circ} 38.21 \mathrm{~W}\right.$; depth $\left.3672 \mathrm{~m}\right)$, and $504\left(1^{\circ} 13.58 \mathrm{~N}, 83^{\circ} 43.93 \mathrm{~W}\right.$; depth $\left.3460 \mathrm{~m}\right)$. The quanti- 


\begin{tabular}{|c|c|c|c|c|c|c|c|c|c|c|c|c|c|c|c|c|c|}
\hline Age & \multicolumn{9}{|c|}{ Late Pliocene } & \multicolumn{8}{|c|}{ Early Pliocene } \\
\hline Zone & \multicolumn{11}{|c|}{ D. stapedia } & \multirow{2}{*}{\multicolumn{6}{|c|}{ D. fibula }} \\
\hline Subzone & \multicolumn{9}{|c|}{ D. ornata } & \multicolumn{2}{|c|}{-} & & & & & & \\
\hline Sub-bottom Depth (m) & ‡̆ & $\check{n}$ & $\begin{array}{l}\text { पे } \\
\stackrel{0}{2}\end{array}$ & $\stackrel{\infty}{\text { i }}$ & $\stackrel{\dddot{5}}{5}$ & $\stackrel{\circ}{\Xi}$ & $\stackrel{\circ}{\varrho}$ & ণ্ণ & $\stackrel{\infty}{\dot{\Xi}}$ & 官 & 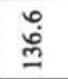 & $\begin{array}{l}\circ \\
\stackrel{\infty}{\oplus}\end{array}$ & 离 & 禹 & $\stackrel{\sim}{\check{n}}$ & $\stackrel{0}{\stackrel{0}{ٌ}}$ & ¿̊․ \\
\hline Taxa & 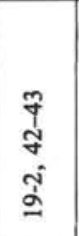 & $\begin{array}{l}\text { ปे } \\
\text { ปे } \\
\text { กิ่ }\end{array}$ & 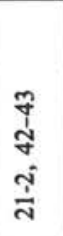 & 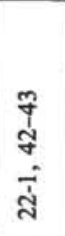 & 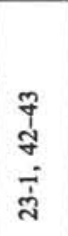 & 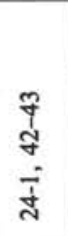 & 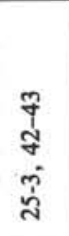 & $\begin{array}{l}\text { yे } \\
\text { भे } \\
\text { के }\end{array}$ & $\begin{array}{l}\text { ปे } \\
\text { ป } \\
\frac{1}{4}\end{array}$ & 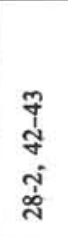 & 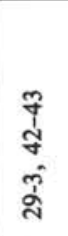 & $\begin{array}{l}\stackrel{m}{+} \\
\text { qे } \\
\text { ì }\end{array}$ & $\begin{array}{l}\frac{m}{y} \\
\text { 岁 } \\
\frac{\vec{m}}{m}\end{array}$ & 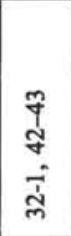 & 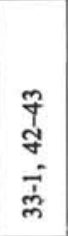 & 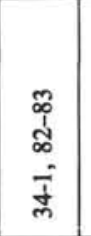 & $\begin{array}{l}\text { y } \\
\text { y } \\
\text { जे } \\
\text { ले }\end{array}$ \\
\hline $\begin{array}{l}\text { Dictyocha sp. aff. } D \text {. aegea } \\
\text { D. angulata } \\
\text { D. aspera } \\
\text { D. brevispina } \\
\text { D. calida ampliata } \\
\end{array}$ & 1 & & $<1$ & & & & & $\begin{array}{r}<1 \\
1\end{array}$ & 1 & $\begin{array}{l}1 \\
1 \\
3\end{array}$ & 3 & $<1$ & $\begin{array}{r}<1 \\
1 \\
1\end{array}$ & 3 & $\begin{array}{l}2 \\
5\end{array}$ & $\begin{array}{l}1 \\
2\end{array}$ & \\
\hline $\begin{array}{l}\text { D. calida calida } \\
D \text {. concavata } \\
D \text {. concinna } \\
D \text {. sp. aff. } D \text {. delicata } \\
D \text {. delicata var. bisecta } \\
\end{array}$ & $\begin{array}{l}<1 \\
<1\end{array}$ & 4 & $\begin{array}{r}5 \\
<1 \\
<1\end{array}$ & $<1$ & & 7 & 1 & 2 & 1 & 3 & & 4 & $<1$ & & 8 & 10 & 2 \\
\hline $\begin{array}{l}\text { D. fibula s. ampl. } \\
\text { D. helix } \\
\text { D. longa } \\
\text { D. longa var. paxilla } \\
\text { D. neonautica var. cocosensis }\end{array}$ & $<1 \mathrm{R}$ & $<1$ & & & & 6 & 9 & $\begin{array}{r}11 \\
<1\end{array}$ & 1 & & $\begin{array}{l}1 \\
3\end{array}$ & $\begin{array}{l}2 \\
3\end{array}$ & $\begin{array}{l}1 \\
4\end{array}$ & $\begin{array}{l}15 \\
38\end{array}$ & $\begin{array}{r}6 \\
22\end{array}$ & \begin{tabular}{c|}
3 \\
4 \\
11 \\
$1 \mathrm{R}$
\end{tabular} & $\begin{array}{l}22 \\
39\end{array}$ \\
\hline $\begin{array}{l}\text { D. ornata africana } \\
D . \text { sp. aff. } D \text {. ornata ornata } \\
\text { D. perfecta } \\
\text { D. perlaevis flexatella } \\
D \text {. perlaevis perlaevis } \\
\end{array}$ & 7 & $\begin{array}{r}<1 \\
2\end{array}$ & $\begin{array}{r}<1 \\
1 \\
\end{array}$ & $\begin{array}{r}12 \\
7 \\
\end{array}$ & 9 & $\begin{array}{r}1 \\
4 \\
7 \\
17 \\
\end{array}$ & $\begin{array}{r}2 \\
<1 \\
20 \\
29 \\
\end{array}$ & $\begin{array}{l}22 \\
14 \\
25\end{array}$ & $\begin{array}{r}8 \\
\\
9 \\
59\end{array}$ & 51 & $\begin{array}{r}4 \\
46 \\
\end{array}$ & $\begin{array}{l}<1 \\
56 \\
\end{array}$ & $\begin{array}{r}8 \\
25 \\
\end{array}$ & $\begin{array}{r}13 \\
<1 \\
10 \\
\end{array}$ & $\begin{array}{r}5 \\
40 \\
\end{array}$ & $\begin{array}{l}23 \\
29 \\
\end{array}$ & 19 \\
\hline $\begin{array}{l}\text { D. pulchella } \\
\text { D. sp. aff. D. pumila } \\
\text { D. stapedia aspinosa } \\
\text { D. sp. aff. D. stapedia aspinosa } \\
\text { D. stapedia stapedia } \\
\end{array}$ & $\begin{array}{r}5 \\
50 \\
\end{array}$ & $\begin{array}{r}9 \\
74 \\
\end{array}$ & $\begin{array}{l}13 \\
73 \\
\end{array}$ & $\begin{array}{r}8 \\
33 \\
\end{array}$ & $\begin{array}{l}62 \\
19 \\
\end{array}$ & $\begin{array}{l}10 \\
13 \\
\end{array}$ & $<1$ & 1 & $\begin{array}{r}<1 \\
1\end{array}$ & $\begin{array}{r}11 \\
5 \\
\end{array}$ & $\begin{array}{r}<1 \\
21 \\
3\end{array}$ & $\begin{array}{r}2 \\
18 \\
1 \\
\end{array}$ & $\begin{array}{l}1 \\
6\end{array}$ & & 1 & & \\
\hline $\begin{array}{l}\text { Daria } \\
\text { D. spp. } \\
\text { Distephanus boliviensis } \\
\text { D. boliviensis (divided ring) } \\
\text { D. crux bispinosus } \\
\end{array}$ & $\begin{array}{l}7 \\
1\end{array}$ & 2 & 2 & $\begin{array}{l}3 \\
3\end{array}$ & 7 & $\begin{array}{l}6 \\
1\end{array}$ & $\begin{array}{r}3 \\
3 \\
<1\end{array}$ & $\begin{array}{l}4 \\
4\end{array}$ & $\begin{array}{l}3 \\
2 \\
3\end{array}$ & $\begin{array}{l}2 \\
5\end{array}$ & $\begin{array}{l}4 \\
2\end{array}$ & $\begin{array}{l}2 \\
3\end{array}$ & $\begin{array}{l}7 \\
2\end{array}$ & 15 & 7 & $\begin{array}{l}1 \\
6 \\
3\end{array}$ & $\begin{array}{r}5 \\
<1 \\
<1 \\
\end{array}$ \\
\hline $\begin{array}{l}\text { D. major } \\
D \text {. sp. aff. D. mesophthalmus } \\
D \text {. quinquangellus } \\
\text { D. speculum bispicatus } \\
D \text {. speculum f. coronata }\end{array}$ & 5 & 4 & 3 & $\begin{array}{r}2 \\
28 \\
<1 \\
\end{array}$ & 1 & $\begin{array}{r}15 \\
1 \\
1 \\
\end{array}$ & 3 & 2 & $\begin{array}{r}<1 \\
1 \\
1 \\
\end{array}$ & $\begin{array}{l}1 \\
3\end{array}$ & $\begin{array}{l}1 \\
2\end{array}$ & $\begin{array}{r}<1 \\
<1 \\
1\end{array}$ & $\begin{array}{l}4 \\
2\end{array}$ & 1 & & 2 & 3 \\
\hline $\begin{array}{l}\text { D. speculum elongatus } \\
\text { D. speculum speculum } \\
\text { D. speculum tenuis } \\
\text { D. sp. A (Bukry, 1979) } \\
\text { D. spp. (cruxoid) } \\
\end{array}$ & $\begin{array}{r}2 \\
19\end{array}$ & 4 & $\begin{array}{l}<1 \\
<1\end{array}$ & $\begin{array}{r}1 \\
<1\end{array}$ & $\begin{array}{r}1 \\
<1\end{array}$ & 10 & $\begin{array}{r}22 \\
<1\end{array}$ & 13 & $\begin{array}{r}<1 \\
5 \\
1\end{array}$ & 11 & $\begin{array}{r}1 \\
<1 \\
\end{array}$ & 6 & $\begin{array}{r}1 \\
15 \\
<1\end{array}$ & $\begin{array}{r}4 \\
<1\end{array}$ & 6 & $\begin{array}{l}7 \\
1\end{array}$ & 9 \\
\hline $\begin{array}{l}\text { Mesocena circulus } \\
\text { M. quadrangula }\end{array}$ & & & & 2 & $<1$ & $<1$ & 1 & 3 & 3 & $\begin{array}{l}3 \\
1 \\
\end{array}$ & 1 & $<1$ & 2 & 1 & 1 & & \\
\hline Total Specimens & 300 & 300 & 300 & 300 & 300 & 300 & 300 & 300 & 300 & 200 & 300 & 300 & 300 & 300 & 200 & 200 & 300 \\
\hline Paleotemperature Value & 74 & 92 & 95 & 64 & 90 & 71 & 70 & 80 & 84 & 76 & 85 & 89 & 74 & 95 & 97 & 87 & 87 \\
\hline
\end{tabular}

Figure 1. (Continued).

tative analysis of silicoflagellates reveals a consistent sequence of appearances and disappearances between some old and new species at all three sites. The sequence within the redefined Miocene-Pliocene Dictyocha fibula Zone includes the disappearance of Dictyocha pulchella just above the Miocene/Pliocene boundary, followed closely by the appearance and acme of $D$. angulata. Both events help define new subzonal boundaries in the ETP between the $D$. angulata Subzone and $D$. pulchella Subzone. According to correlative diatom events and age 


\section{Cores 3 to 18}

\begin{tabular}{|c|c|c|c|c|c|c|c|c|c|c|c|c|c|c|c|c|c|}
\hline Age & $\begin{array}{c}\text { Zone } \\
\text { (Subzone) }\end{array}$ & $\begin{array}{c}\text { Hole } 504 \\
\text { Sample } \\
\text { (interval in } \mathrm{cm} \text { ) }\end{array}$ & $\begin{array}{c}\text { Sub- } \\
\text { bottom } \\
\text { Depth } \\
\text { (m) }\end{array}$ & 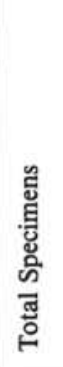 & 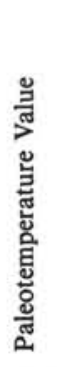 & 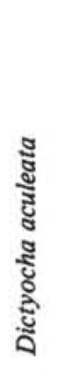 & 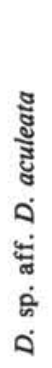 & $\begin{array}{l}\text { ปีँ } \\
\text { जั } \\
\text { है } \\
\text { व }\end{array}$ & 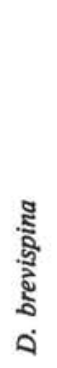 & 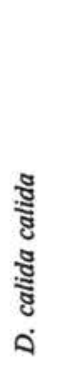 & 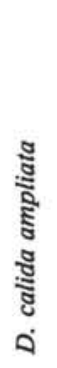 & 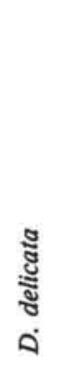 & 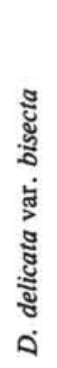 & 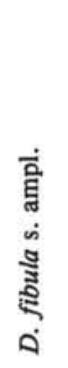 & $\begin{array}{l}\dddot{z} \\
\vdots \\
\vdots\end{array}$ & $\begin{array}{l}\stackrel{0}{0} \\
\text { ఏ } \\
0\end{array}$ & 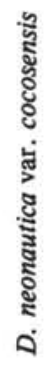 \\
\hline \multirow[b]{2}{*}{$\begin{array}{l}\text { Pleisto- } \\
\text { cene }\end{array}$} & D. aculeata & $\begin{array}{l}3-3,42-43 \\
4, C C \\
5-2,42-43 \\
5, C C \\
\end{array}$ & $\begin{array}{l}22.2 \\
27.4 \\
29.5 \\
31.2 \\
\end{array}$ & $\begin{array}{l}200 \\
200 \\
300 \\
200 \\
\end{array}$ & $\begin{array}{l}98 \\
97 \\
95 \\
94 \\
\end{array}$ & $\begin{array}{r}6 \\
13 \\
9 \\
12 \\
\end{array}$ & $\begin{array}{r}1 \\
4 \\
15 \\
9 \\
\end{array}$ & & & $\begin{array}{r}36 \\
10 \\
7\end{array}$ & $\begin{array}{l}1 \\
1\end{array}$ & 1R & & 2 & & $1 \mathrm{R}$ & IR \\
\hline & M. quadrangula & $\begin{array}{l}6-1,42-43 \\
6-2,42-43 \\
7-2,42-43 \\
8-2,42-43 \\
9-2,42-43 \\
10-2,42-43 \\
11-2,42-43 \\
\end{array}$ & $\begin{array}{l}32.4 \\
33.9 \\
38.3 \\
42.7 \\
47.1 \\
51.5 \\
55.9 \\
\end{array}$ & $\begin{array}{l}300 \\
300 \\
300 \\
300 \\
300 \\
300 \\
300 \\
\end{array}$ & $\begin{array}{l}96 \\
98 \\
84 \\
63 \\
65 \\
68 \\
68 \\
77 \\
\end{array}$ & $\begin{array}{l}3 \\
9\end{array}$ & $\begin{array}{l}3 \\
5\end{array}$ & 更 & 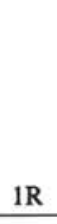 & $\begin{array}{r}<1 \\
3 \\
13 \\
1 \\
<1 \\
\\
2\end{array}$ & $\begin{array}{l}1 \\
1 \\
1 \\
1\end{array}$ & & & & & & \\
\hline $\begin{array}{c}\text { Late } \\
\text { Pliocene }\end{array}$ & $\begin{array}{l}\text { D. stapedia } \\
\text { (D. delicata) }\end{array}$ & $\begin{array}{l}12-1,22-23 \\
13-3,42-43 \\
14-2,42-43 \\
15-1,112-113 \\
16-2,42-43 \\
17-1,42-43 \\
18-3,42-43\end{array}$ & $\begin{array}{l}58.6 \\
66.2 \\
69.1 \\
72.7 \\
77.9 \\
80.8 \\
88.2\end{array}$ & $\begin{array}{l}300 \\
300 \\
200 \\
300 \\
300 \\
300 \\
300\end{array}$ & $\begin{array}{l}91 \\
90 \\
78 \\
87 \\
88 \\
69 \\
91\end{array}$ & & & & $\begin{array}{l}1 \\
1\end{array}$ & $\begin{array}{r}1 \\
1 \\
2 \\
1 \\
43\end{array}$ & $\begin{array}{r}<1 \\
1\end{array}$ & $\begin{array}{r}9 \\
29 \\
6 \\
23\end{array}$ & IR & $\begin{array}{r}1 \\
<1\end{array}$ & $\begin{array}{l}\mathrm{X} \\
<1\end{array}$ & & \\
\hline
\end{tabular}

Figure 1. (Continued).

estimates at DSDP Holes 503A and 504, the last common occurrence of $D$. pulchella is bracketed between the highest occurrence surface (HOS) of Thalassiosira miocenica and the lowest occurrence surface (LOS) of Nitzschia jouseae ( 4.4-5.0 Ma). The D. pulchella event occurs between Core 47 and 45 of DSDP Hole 504.

The first Dictyocha angulata s. str. is in Core 42 at DSDP Hole 504, which is bracketed below by diatom HOS Nitzschia cylindrica and above by LOS Thalassiosira convexa $(\sim 3.5-4.2 \mathrm{Ma})$ at DSDP Holes $503 \mathrm{~A}$ and 504. D. angulata has significant abundances up to $30 \%$, or consistent presence, through Cores 36 to 42 of DSDP Hole 504. Compared specimens of D. angulata appear slightly lower in Cores 44 and 45 ; sparse, sporadic, possibly displaced specimens occur between Cores 28 to 34 in the lower Pliocene.

The next-higher event at DSDP Holes 495, 503A, and 504 is the brief appearance of Dictyocha delicata var. bisecta, which is in the lowest Dictyocha stapedia Zone of DSDP Holes 495 and 503A, but in the highest Dictyocha fibula Zone of DSDP Hole 504. All occurrences are near the upper/lower Pliocene boundary, DSDP Hole 504 occurrences being the oldest. D. delicata var. bisecta is bracketed at both DSDP Holes 503A and 504 by the diatom events LOS Thalassiosira convexa s. str. below and LOS Rhizosolenia praebergonii above ( $~ 3.0-3.5$ Ma).
This comparison of significant silicoflagellate and diatom events between ETP sites shows divergence of the first Dictyocha stapedia stapedia from the diatom age curve of DSDP Holes 503A and 504. This may be a result of the sparseness of early occurrences and of the interpretation of possible transitional specimens in early populations. Note the overlapping occurrences between $D$. longa var. paxilla and $D$. stapedia stapedia near the upper/lower boundary at DSDP Hole 503A (Bukry, in press a). Allowing for the abbreviated stratigraphic coverage at DSDP Hole 495, however, the general sequence near the boundary includes Dictyocha delicata var. $b i$ secta acme below, followed closely by first $D$. stapedia stapedia s. str., and finally at or just above the boundary the appearances of $D$. ornata africana and $D$. perlaevis flexatella. The distinctive morphologies of the latter two subspecies are convenient guides to distinguish upper from lower Pliocene silicoflagellate assemblages at these DSDP sites in the ETP.

The basal subzone of the upper Pliocene, Dictyocha ornata Subzone, occurs at both DSDP Holes 503A and 504. The brief acme of new species Dictyocha concinna has been recorded in single Cores 503A-13 and 504-24 near the top of the range of $D$. longa. These occurrences of $D$. concinna coincide with the diatom age-depth curve between HOS Nitzschia jouseae, below, and HOS Thalassiosira convexa, above ( 2.1-2.5 Ma). 


\begin{tabular}{|c|c|c|c|c|c|c|c|c|c|c|c|c|c|c|c|c|c|c|c|c|c|}
\hline 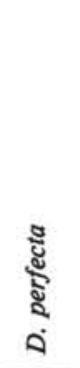 & 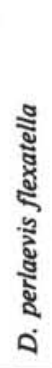 & 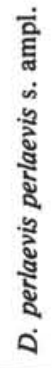 & 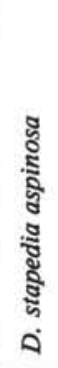 & 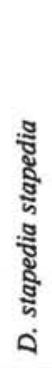 & 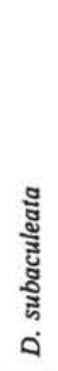 & 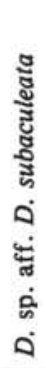 & 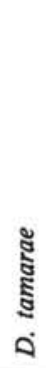 & 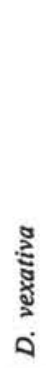 & 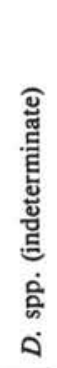 & 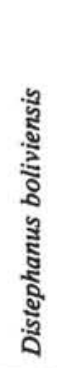 & 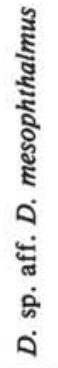 & 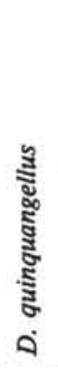 & 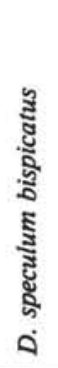 & 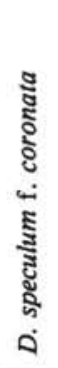 & 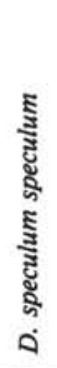 & 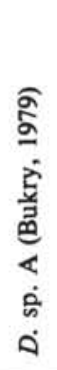 & 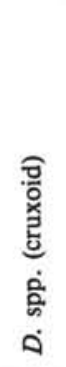 & 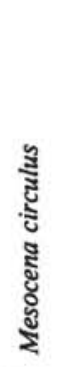 & 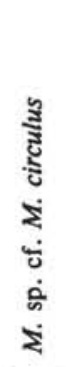 & 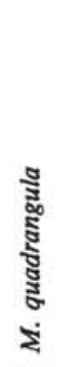 & 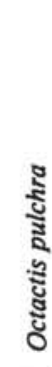 \\
\hline & & $\begin{array}{r}18 \\
8 \\
4 \\
25 \\
\end{array}$ & $\begin{array}{r}5 \\
32 \\
1 \\
14 \\
\end{array}$ & $\begin{array}{r}26 \\
12 \\
41 \\
6 \\
\end{array}$ & $\begin{array}{r}7 \\
7 \\
17 \\
25 \\
\end{array}$ & & & & $\begin{array}{r}1 \\
10 \\
6 \\
\end{array}$ & & & & & & 2 & $\begin{array}{l}3 \\
2 \\
\end{array}$ & & & $<1$ & & $\begin{array}{l}2 \\
1 \\
1 \\
4 \\
\end{array}$ \\
\hline & & $\begin{array}{r}61 \\
58 \\
38 \\
7 \\
1 \\
2 \\
7 \\
\end{array}$ & $\begin{array}{r}18 \\
<1 \\
1\end{array}$ & $\begin{array}{r}6 \\
13 \\
12 \\
12 \\
27 \\
30 \\
42 \\
\end{array}$ & $\begin{array}{r}3 \\
3 \\
4 \\
<1\end{array}$ & $\begin{array}{l}4 \\
4 \\
\end{array}$ & & & $\begin{array}{r}3 \\
4 \\
1 \\
1 \\
<1 \\
1 \\
1 \\
\end{array}$ & $\begin{array}{l}<1 \\
<1\end{array}$ & & & $\begin{array}{r}<1 \\
1 \\
\end{array}$ & & $\begin{array}{r}<1 \\
1 \\
1 \\
\end{array}$ & $\begin{array}{l}2 \\
1 \\
1\end{array}$ & & & & $\begin{array}{r}4 \\
4 \\
31 \\
73 \\
68 \\
58 \\
40 \\
\end{array}$ & $\begin{array}{r}1 \\
1 \\
<1 \\
<1 \\
<1 \\
\\
2 \\
\end{array}$ \\
\hline$<1 R$ & $\begin{array}{l}1 \\
\mathrm{X}\end{array}$ & $\begin{array}{r}8 \\
25 \\
8 \\
28 \\
10 \\
9 \\
23\end{array}$ & $\begin{array}{r}1 \\
1 \\
1 \\
10 \\
<1\end{array}$ & $\begin{array}{l}67 \\
27 \\
62 \\
31 \\
39 \\
44 \\
12\end{array}$ & & 3 & $\begin{array}{r}30 \\
1\end{array}$ & 1 & $\begin{array}{r}2 \\
<1 \\
5 \\
1\end{array}$ & $\begin{array}{r}<1 \\
3\end{array}$ & 9 & $\begin{array}{l}5 \\
6 \\
5 \\
7 \\
1\end{array}$ & $\begin{array}{r}<1 \\
1 \\
<1\end{array}$ & 1 & $\begin{array}{r}1 \\
<1 \\
5 \\
2 \\
<1 \\
2 \\
<1\end{array}$ & $\begin{array}{r}<1 \\
5 \\
6 \\
5 \\
6 \\
23 \\
1\end{array}$ & $\begin{array}{r}1 \\
<1\end{array}$ & 1 & & $\begin{array}{r}14 \\
11 \\
2\end{array}$ & 1 \\
\hline
\end{tabular}

Figure 1. (Continued).

Similarities in the Pliocene silicoflagellate sequences of events for the ETP DSDP sites, especially the two hydraulic-piston-cored sites, indicates that local biostratigraphic correlation using silicoflagellates is possible, and that considerable improvement can be expected when taxonomic discrimination is refined.

\section{SYSTEMATIC PALEONTOLOGY OF NEW TAXA}

\section{Genus DICTYOCHA Ehrenberg, 1837 \\ Dictyocha concinna Bukry, n. sp.}

(Plate 2, Figs. 5-11)

Dictyocha sp. A Bukry, in press c, pl. 5, fig. 3.

Description. Dictyocha concinna has a moderate-sized ring that is only very slightly scalloped and nearly square in format; the minor axis internal diameter is 93 to $98 \%$ ( $96 \%$ mean) of the major axis. All spines are short and essentially equant. Basal pikes are short and are under or just beside the strut-ring junctions. The bar is short and the struts are oriented symmetrically.

Remarks. Dictyocha concinna is distinguished from $D$. calida calida by much shorter spines, shorter and thicker bar, less-scalloped outline, more-equant axial diameters yielding a squarer format. $D$. calida calida axial ratios range from 87 to $93 \%$ (mean $91 \%$ ). Shorter spines and the squarer format are most distinctive. D. concinna is distinguished from $D$. stapedia aspinosa by symmetric strut orientation and short spines.

Occurrence. Dictyocha concinna was first tabulated and illustrated from a single upper Pliocene sample at DSDP Hole 503A, northwest of the Islas Galapagos (Bukry, in press a). The abundance of 5\% and short range in the Dictyocha ornata Subzone justified distinction from " $D$. spp." At DSDP Hole 504, this identical species occurs at $7 \%$ in a single sample in the $D$. ornata Subzone. In addition, the $D$. concinna occurrences from DSDP Holes 503A and 504 plot onto the diatom age curve for these two sites at a level between HOS of Nitzschia jouseae and HOS of Thalassiosira convexa $(\sim 2.1-2.5 \mathrm{Ma})$. This suggests that D. concinna provides a new marker horizon within the upper Pliocene D. ornata Subzone of the ETP.

Size. Maximum internal diameter 19 to $28 \mu \mathrm{m}$ (holotype $22 \mu \mathrm{m}$ ). Holotype. USNM 321351 (Plate 2, Fig. 5).

Isotypes. USNM 321352 to 321356.

Type locality. Eastern tropical Pacific, DSDP Sample 504-24-1, $42-43 \mathrm{~cm}$.

Dictyocha helix Bukry, n. sp.

(Plate 3, Figs. 5-10; Plate 4, Figs. 1, 2)

Description. Dictyocha helix has a strongly canted, short apical bar which has a spire on some specimens. The strut-ring junctions are nonsymmetric. The basal-ring pattern is slightly torqued in a clockwise sense in apical view, as seen by nonalignment of spine sets and ring curvature. Spines are moderate to typically long, but basal pikes are missing or very small, directly below the struts. The minor-axis inner diameter is 92 to $100 \%$ (mean $94 \%$ ) of the major axis, producing nearly equant rings. Bar orientation is about equally divided between asperoid and fibuloid forms.

Remarks. Dictyocha helix is distinguished from the similarly torqued subspecies $D$. perlaevis flexatella by much shorter bar and squarer, less-elongate ring format, resulting in struts that are more equant. $D$. helix is distinguished from older $D$. aspera clinata by shorter bar and thinner spines.

Occurrence. Dictyocha helix is most abundant $(1 \%)$ in the upper Pliocene of Core 19 at DSDP Hole 504. It is sparser above and below in Cores 18 and 20. This range straddles the Dictyocha ornata Subzone/ $D$. delicata Subzone boundary.

Size. Maximum internal diameter 20 to $24 \mu \mathrm{m}$ (holotype $22 \mu \mathrm{m}$ ).

Holotype. USNM 321357 (Plate 3, Figs. 5,6).

Isotypes. USNM 321358 to 321362 .

Type locality. Eastern tropical Pacific, DSDP Sample 504-19-2, $42-43 \mathrm{~cm}$. 


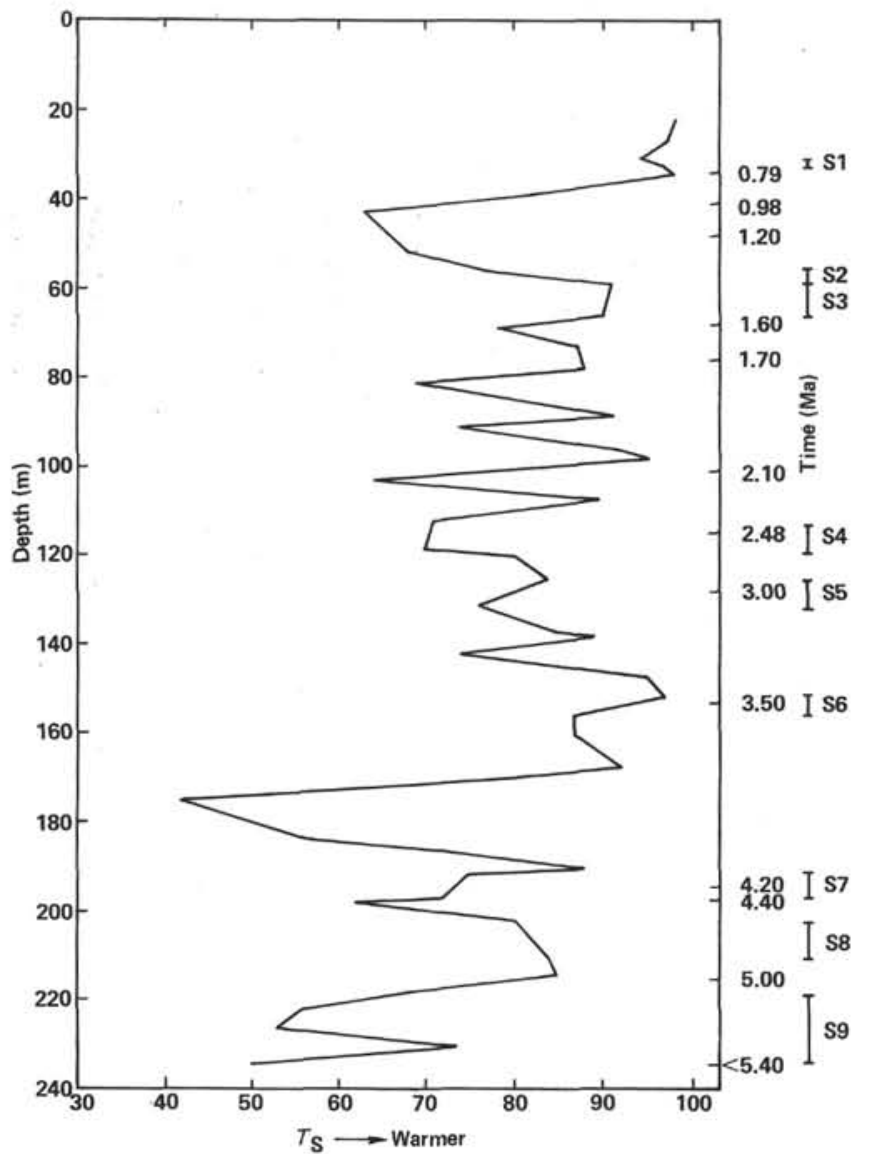

Figure 2. Silicoflagellate relative paleotemperature curve for DSDP Hole 504 samples. Approximate chronology based on shipboard diatom ages (Ma); see also Sancetta (this volume). Silicoflagellate data for the curve are available in the species-occurrence figures. Useful silicoflagellate biostratigraphic events include: $\mathrm{S} 1=\mathrm{Last}$ Mesocena quadrangula. S2 = Last Dictyocha delicata. S3 = First Octactis pulchra. S4 = First Dictyocha concinna . S5 = First Dictyocha ornata africana. S6 = First Dictyocha delicata var. bisecta . S7 = First Dictyocha angulata . S8 = Last common consistent occurrence of Dictyocha pulchella. S9 = Local late Miocene acme of Mesocena quadrangula.

Dictyocha tamarae Bukry, n. sp.

(Plate 5, Figs. 7-11; Plate 6, Fig. 1)

Description. Dictyocha tamarae haš a large ring with fairly angular corners, especially along the major axis. Basal pikes are long and conspicuous; one opposed set is at strut-ring junctions and the other well offset, between the strut junction and minor axis. The bar is very long and may have a low spire. The width of the bar equals that of the ring or is very slightly narrower. Struts are short and oriented slightly asymmetrically. Spines are moderate, and the major-axis spines are longer than the minor-axis spines.

Remarks. Dictyocha tamarae is distinguished from $D$. delicata var. bisecta by more-equant axial lengths and more-angular basal ring. It is also distinguished by conspicuous basal pikes, spire, and thicker bar. Both have a prominent long bar as a main characteristic. D. tamarae is distinguished from $D$. delicata by longer and thicker bar, longer basal pikes, more-angular ring apices, and less-scalloped outline.

Occurrence. Dictyocha tamarae occurs in the upper Pliocene Cores 16 and 17 of DSDP Hole 504 at abundances of 1 and 30\%. These occurrences are within the Dictyocha delicata Subzone, but $D$. delicata is missing in these cores, and $D$. tamarae seems to have taken its place.

Size. Maximum internal diameter 25 to $30 \mu \mathrm{m}$ (holotype $27 \mu \mathrm{m}$ ).

Holotype. USNM 321363 (Plate 5, Figs. 7,8).

Isotypes. USNM 321364 to 321367.

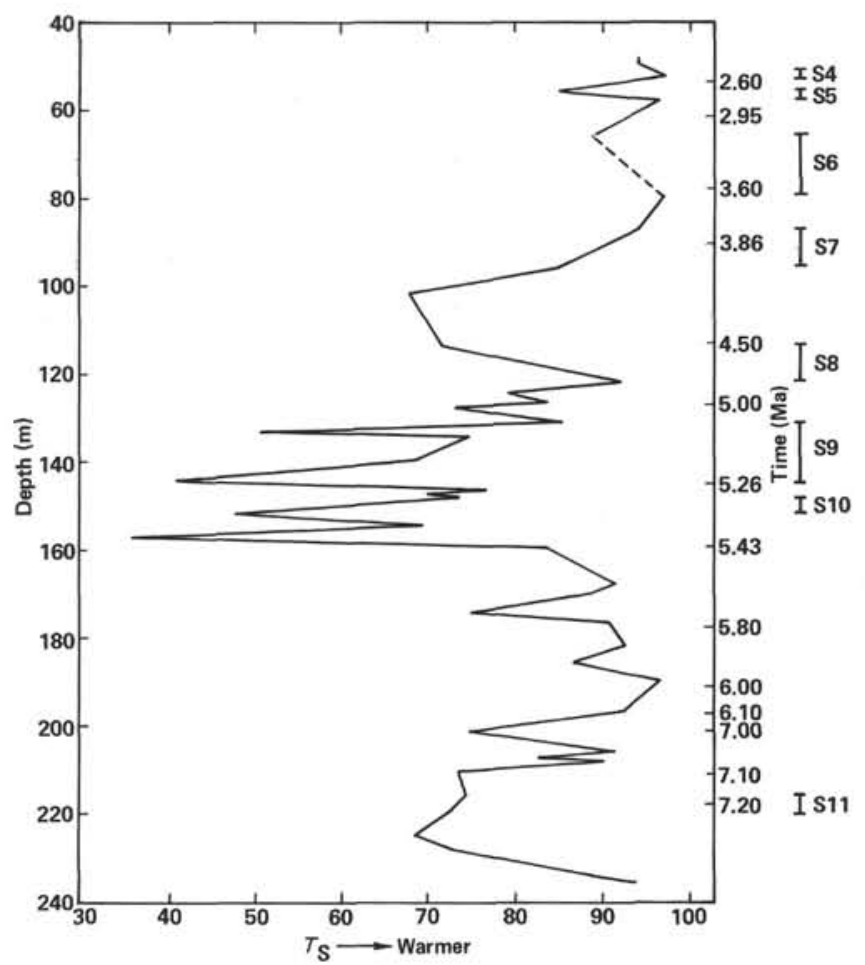

Figure 3. Silicoflagellate relative paleotemperature curve for DSDP Hole 503A samples. Approximate chronology based on shipboard diatom ages (Ma); see also Sancetta (this volume). Silicoflagellate data for the curve are available in the original species-occurrence figures (Bukry, in press a). Useful silicoflagellate biostratigraphic events include: $\mathrm{S} 10=$ First Dictyocha neonautica var. cocosensis. $\mathrm{S} 11$ = First Dictyocha longa . See Figure 2 for definitions of S1 to S9.

Type locality. Eastern tropical Pacific, DSDP Sample 504-17-1, $42-43 \mathrm{~cm}$.

\section{Dictyocha sp. A}

(Plate 6, Figs. 5-8)

Remarks. During photography, one of the silicoflagellates tabuated among the unspecified Dictyocha spp. from Sample 504-44-1, $42-43 \mathrm{~cm}$ was distinguished by small size, short spines, short bar, and great width along the minor axis. Dictyocha sp. A is distinguished from $D$. concinna by smaller size and less-equant spines, and by a more-inflated appearance along the minor axis, which is created by great width and more-rounded portals. Size and shape are consistent within the sample. Maximum internal diameter is 16 to $18 \mu \mathrm{m}$.

\section{SYSTEMATIC PALEONTOLOGY OF PREVIOUSLY CITED TAXA}

\section{Genus DICTYOCHA Ehrenberg, 1837}

Dictyocha aculeata (Lemmermann) Dumitrică

Dictyocha fibula var. aculeata Lemmermann, 1901, p. 261, pl. 11, figs. 1,2 .

Dictyocha aculeata (Lemmermann) Dumitrică, 1973a, p. 907, pl. 9, figs. 5-10.

Remarks. Many of the forms with affinities to Dictyocha aculeata have a long apical bar.

\section{Dictyocha aegea Stradner and Bachmann}

Dictyocha aegea Stradner and Bachmann, 1978, p. 805, pl. 1, figs. 1216; fig. 1.

Dictyocha venzoi Morlotti and Rio, 1978 [1980], p. 102, pl. 2, figs. 5, 6; pl. 3, fig. 1 .

Dictyocha aegea Stradner and Bachmann, Bukry, in press c. 
Remarks. Forms with affinities to Dictyocha aegea in Cores 27 and 28 are elongate but lack the typical recurved side portals (Plate 1, Fig. 1).

\section{Dictyocha angulata Bukry}

(Plate 1, Figs. 2-5)

Dictyocha angulata Bukry, in press c, pl. 1, figs. 9-12.

Remarks. Some aberrant specimens lacking minor-axis spines and some having asperoid bar alignment occur in the highest sample (Core 36), where Dictyocha angulata is abundant. In Core 44 , below the range of $D$. angulata s. str., some specimens intermediate between $D$. fibula and $D$. angulata are tabulated as $D$. sp. cf. $D$. angulata. The high abundance of $D$. angulata near the top of the Dictyocha fibula Zone in Cores 36 to 42 aids in distinguishing the new Dictyocha angulata Subzone.

\section{Dictyocha aspera aspera (Lemmermann) Bukry and Foster}

Dictyocha fibula var. aspera Lemmermann, 1901, p. 260, pl. 10, figs. 27,28 .

Dictyocha aspera (Lemmermann) Bukry and Foster, 1973 (in part), p. 826 , pl. 2 , figs. 4,6 .

\section{Dictyocha aspera clinata Bukry}

Dictyocha clinata aspera Bukry, 1975, p. 695, pl. 1, figs. 1-5.

\section{Dictyocha brevispina (Lemmermann) Bukry}

(Plate 1, Figs. 6-11; Plate 2, Figs. 1, 2)

Dictyocha fibula var. brevispina Lemmermann, 1901, p. 260

Dictyocha brevispina (Lemmermann) Bukry, 1976, p. 723.

Dictyocha brevispina (Lemmermann), Bukry, in press d.

Remarks. Variant populations without bars (Core 45) and giant asymmetric forms are tabulated separately. Dumitrică (1973b) illustrated a barless variant at DSDP Hole 206 from lower Pliocene Core 18.

\section{Dictyocha calida ampliata Bukry}

(Plate 2, Fig. 4)

Dictyocha calida ampliata Bukry, 1979a, p. 982, pl. 2, figs. 1,2,9.

Remarks. One specimen with all portals elongate and a short bar was noted in Sample 504-5-2, 42-43 cm.

\section{Dictyocha calida calida Poelchau}

Dictyocha calida Poelchau, 1976, p. 169, pl. 1, figs. a-f.

Dictyocha calida calida Poelchau, Bukry, 1979a, p. 982, pl. 2, figs. 3,4 .

\section{Dictyocha concavata Dumitrică}

(Plate 2, Fig. 3)

Dictyocha concavata Dumitrică, 1978, p. 212, pl. 3, figs. 1-4.

Dictyocha concavata Dumitrică, Bukry, in press d, pl. 1, figs. 1,2.

\section{Dictyocha delicata (Bukry) Bukry \\ (Plate 3, Fig. 1)}

Dictyocha perlaevis delicata Bukry, 1976, p. 724, pl. 1, figs. 5-10. Dictyocha delicata (Bukry) Bukry, in press c, pl. 2, fig. 7.

Remarks. Dictyocha delicata is common only in late Pliocene and early Pleistocene Cores 12 to 18 . Sparse $D$. sp. aff. $D$. delicata in lower cores have thin apical bars, but the ring is not proportionally as thick as in $D$. delicata. A new, possibly related species, $D$. tamarae, is distinguished by its long bar and angular ring; $D$. delicata var. bisecta has a long bar, but a rounded ring.

\section{Dictyocha delicata (Bukry) Bukry var. bisecta Bukry}

(Plate 3, Figs. 2-4)

Dictyocha delicata (Bukry) Bukry var. bisecta Bukry, in press c, pl. 2, figs. 3-6.

Remarks. This distinctive variety with an unscalloped ring occurred in a single sample at DSDP Hole 495, to the north, in the base of the Dictyocha stapedia Zone. At DSDP Hole 504, it occurs slightly lower, in the uppermost $D$. fibula Zone. The earliest population of Dictyocha delicata var. bisecta in Core 33 of DSDP Hole 504 contains several specimens with robust rings that suggest similarity to the form of Mesocena quadrangula. This species could be a source for polyphyletic M. quadrangula.

\section{Dictyocha fibula Ehrenberg}

Dictyocha fibula Ehrenberg, 1839, fide Loeblich et al., 1968, p. 90, pl. 9, figs. 7-12.

Dictyocha fibula fibula Ehrenberg, Bukry, 1976, p. 723, pl. 1, figs. 3,4 .

Dictyocha fibula fibula Ehrenberg, Bukry, 1978b, p. 697, pl. 2, figs. 1,2 .

Dictyocha fibula Ehrenberg, Bukry, 1980a, p. 514, pl. 2, figs. 1,2.

Remarks. Moderate to small forms with little scalloping of the ring are tabulated as Dictyocha fibula s. ampl. The morphologic pattern of this species is simple, and direct lineages to the original middle and upper Miocene populations illustrated from Ehrenberg in Loeblich et al. (1968, pl. 9) would be difficult. Figures 8 and 9 (especially) in the Loeblich reference are used for the species concept of $D$. fibula. Figure 12 is similar, but seems to have asymmetric strut placement, instead of the regular arrangement in $D$. fibula of Figure 9.

\section{Dictyocha longa Bukry \\ (Plate 4, Figs. 3,4)}

Dictyocha perlaevis perlaevis Frenguelli, Bukry, 1980b (in part), p. 553 , pl. 4, figs. 10-13.

Dictyocha longa Bukry, in press c, pl. 2, figs. 8-13; pl. 3, figs. 1,2.

Remarks. A few specimens of Dictyocha longa in Core 31 lack minor-axis spines, otherwise the morphology is similar to forms at other ETP sites.

\section{Dictyocha longa var. paxilla Bukry}

(Plate 4, Figs. 5,6)

Dictyocha longa var. paxilla Bukry, in press c, pl. 3, figs. 3-8. Dictyocha longa var. paxilla Bukry, Bukry, in press a, pl. 2, fig. 9.

\section{Dictyocha neonautica var. cocosensis Bukry}

(Plate 4, Fig. 7)

Dictyocha neonautica var. cocosensis Bukry, in press b.

Dictyocha navicula Ehrenberg, Bukry and Foster, 1973 (in part), p. 827 , pl. 3 , fig. 8 .

Dictyocha neonautica var. cocosensis Bukry, in press d, pl. 3, figs. 1-3.

Remarks. Most of the strata cored at DSDP 504 are younger than the main range of Dictyocha neonautica var. cocosensis. The specimen in the basal part of Core 53 probably is the top of the natural range, but it could also be mixed from below, because shipboard scientists described intense bioturbation of Core 53 .

\section{Dictyocha ornata ornata (Bukry) Bukry}

Dictyocha perlaevis ornata Bukry, 1977, p. 922, pl. 1, figs. 1-6. Dictyocha ornata ornata (Bukry) Bukry, in press c.

Remarks. Dictyocha sp. aff. D. ornata ornata is less abundant than D. ornata africana at DSDP Hole 504, and occurs in the upper part of the Dictyocha ornata Subzone. These larger-than-normal specimens are recorded in Cores 19 to 21 .

\section{Dictyocha ornata africana Bukry}

Dictyocha perlaevis ornata Bukry, Bukry, 1978a, p. 644, pl. 1, figs. 7,8 .

Dictyocha ornata africana Bukry, in press c, pl. 3, fig. 11; pl. 4, figs. 1,2 .

Remarks. Dictyocha ornata africana occurs earlier and more abundantly than $D$. ornata ornata at DSDP Hole 504 in upper Pliocene Cores 24 to 27 .

\section{Dictyocha perfecta Bukry}

(Plate 4, Figs. 8,9)

Dictyocha perfecta Bukry, in press a, pl. 3, figs. 5-10.

Remarks. Typically formed Dictyocha perfecta are common in Pliocene Cores 26 and 32. D. perfecta is a large species, distinguished from $D$. perlaevis by both narrower format and shorter bar. 


\section{Dictyocha perlaevis flexatella Bukry}

(Plate 4, Figs. 10,11)

Dictyocha perlaevis flexatella Bukry, 1979a, p. 984, pl. 3, figs. 1-3. Remarks. Dictyocha perlaevis flexatella is limited to the upper Pliocene at DSDP Hole 504. Fully-developed forms occur with those showing partial torque symmetry.

\section{Dictyocha perlaevis perlaevis Frenguelli \\ (Plate 4, Fig. 12)}

Dictyocha perlaevis Frenguelli, 1951, p. 279, figs. 4b, c.

Dictyocha perlaevis perlaevis Frenguelli, Bukry, 1979a, p. 984, pl. 3, figs. 6-11.

Remarks. Dictyocha perlaevis perlaevis is a common to abundant warm-water species which attains its greatest abundance $(59 \%)$ at DSDP Hole 504 (Core 27) in the middle of the Pliocene. Specimens in Core 17 have a shorter bar than is typical.

\section{Dictyocha pulchella Bukry}

(Plate 5, Fig. 1)

Dictyocha pulchella Bukry, 1975, p. 687, pl. 4, figs. 1-3.

Remarks. Dictyocha pulchella is smaller than D. brevispina and has relatively larger minor-axis portals. It is common to abundant from the upper lower Miocene to lower Pliocene. It is essentially absent above Core 47 , which marks the top of the new $D$. pulchella Subzone of the Dictyocha fibula Zone.

Some specimens from Cores 52 and 53 have longer bars and moreangular short-axis portals than typical $D$. pulchella (Plate 5, Fig. 2); these are tabulated separately.

\section{Dictyocha pumila (Ciesielski) Bukry}

Dictyocha fibula var. pumila Ciesielski, 1975, p. 656, pl. 5, figs. 5-10; pl. 6, fig. 1, ?2.

Dictyocha pumila (Ciesielski) Bukry, 1978a, p. 642.

Dictyocha pumila (Ciesielski), Bukry, 1979a, p. 984, pl. 4, fig. 3.

Remarks. A few small lower Pliocene specimens in Cores 29 to 31 show flexed rings and slightly bent spines and resemble Dictyocha pumila. A population of $D$. sp. aff. D. stapedia aspinosa in Core 23 is suggestive of $D$. pumila, but these specimens have only slightly irregular rings and normal spines.

\section{Dictyocha stapedia aspinosa Bukry}

Dictyocha stapedia aspinosa Bukry, 1976, p. 724, pl. 2, figs. 6-9.

Remarks. This moderate-sized taxon ranges from the lower Pliocene to upper Pleistocene at abundances up to $32 \%$ at DSDP Hole 504. An unusual population with slightly irregular rings is tabulated as D. sp. aff. D. stapedia aspinosa for Core 23 (Plate 5, Fig. 3).

\section{Dictyocha stapedia stapedia Haeckel}

Dictyocha stapedia Haeckel, 1887, p. 1561, pl. 101, figs. 10-12. Dictyocha stapedia stapedia Haeckel, Bukry, 1980b, p. 553, pl. 5, figs. 8-10.

Remarks. On the basis of DSDP Hole 503A assemblages, early Dictyocha stapedia stapedia appears to be derived from D. longa var. paxilla through at least one transitional form with lobed outline, but asymmetric struts. Such a transition form was not observed from DSDP Hole 504, and, aside from the early form in Cores 29 and 30, the other populations have typical morphologies.

D. stapedia stapedia is one of the dominant species through the upper Pliocene to Pleistocene in Core 24 and above.

\section{Dictyocha subaculeata (Bukry) Bukry}

Dictyocha aculeata subaculeata Bukry, 1980b, p. 552, pl. 1, figs. 8-17. Dictyocha subaculeata (Bukry) Bukry, in press c.

Remarks. Dictyocha sp. aff. D. subaculeata in Cores 10 and 11 are large like $D$. aculeata, but lack the diagnostic peripheral pikes of that species (Plate 5, Fig. 4).

\section{Dictyocha subclinata Bukry}

Dictyocha subclinata Bukry, in press d, pl. 1, figs. 4-8; pl. 2, figs. $1-10$.

Remarks. Only specimens with close affinities to Dictyocha subclinata (Plate 5, Figs. 5,6) were encountered in Core 53.

\section{Dictyocha transenna Bukry}

(Plate 6, Fig. 2)

Dictyocha transenna Bukry, in press a, pl. 4, figs. 1-12; pl. 5, figs. 1,2. Remarks. One specimen of this late Miocene species is possibly displaced in earliest Pliocene Core 48. Sparse specimens bearing minoraxis spines occur in late Miocene Cores 52 and 53 and are tabulated as $D$. sp. aff. D. transenna (Plate 6, Figs. 3,4). It is not clear whether the extra spines are a recapitulation of the parent stock or a new, reiterative development in $D$. transenna.

\section{Dictyocha varia Locker}

Dictyocha varia Locker, 1975 , p. 7 , text-figs. $1 / 1$ to $1 / 3$ and 3 to 7 .

\section{Dictyocha vexativa Bukry}

Dictyocha vexativa Bukry 1978a, p. 642, pl. 1, figs. 10-14.

Dictyocha spp.

(Plate 6, Figs. 9-12)

Remarks. This is a miscellaneous group that is largely composed of tilted or covered specimens and presently unclassified morphologies. The vast majority are fibuloid specimens which cannot be assigned with assurance to any currently used taxonomic concept.

\section{Genus DISTEPHANUS Stöhr, 1880}

Distephanus boliviensis (Frenguelli) Bukry and Foster

(Plate 7, Fig. 1)

Dictyocha boliviensis Frenguelli, 1940, p. 44, fig. 4 .

Distephamus boliviensis (Frenguelli) Bukry and Foster, 1973, p. 827, pl. 4, figs. 1-3.

Remarks. Specimens with the divided apical rings, but having the same basal ring and spine configuration of Distephanus boliviensis, are not assigned to $D$. major. They occur in upper Pliocene Cores 25 and 27 and are tabulated as D. boliviensis (divided ring).

\section{Distephanus crux crux (Ehrenberg) Haeckel}

Dictyocha crux Ehrenberg, 1840, p. 207; Ehrenberg, 1854, pl. 18, fig. 56; pl. 20 (1), fig. 46; pl. 33 (15), fig. 9; pl. 33 (16), fig. 9; pl. 33 (17), fig. 5.

Distephanus crux (Ehrenberg), Bukry, in press c.

\section{Distephanus crux bispinosus Dumitrică}

(Plate 7, Fig. 2)

Distephanus crux spp. bispinosus Dumitrică, 1973b, p. 850, pl. 6, figs. 3,6,7.

Remarks. Distephanus crux bispinosus is sparse in lower Pliocene Cores 35 to 48 at DSDP Hole 504 .

\section{Distephanus crux carolae Bukry}

(Plate 7, Fig. 3)

Distephanus crux (Ehrenberg) Haeckel subsp. carolae Bukry, in press a, pl. 5, figs. 4-11.

Remarks. Distephanus crux carolae is sparse in the lower Pliocene and upper Miocene at DSDP Hole 504. This extends its upper Miocene range at DSDP Hole 503A.

\section{Distephanus frugalis (Bukry) Bukry}

Distephanus boliviensis frugalis Bukry, 1975, p. 688, pl. 2, figs. 2-7. Distephanus frugalis (Bukry) Bukry, 1979b, p. 561, pl. 3, figs. 5,6.

Remarks. Only sparse compared specimens occur between Cores 40 and 49.

\section{Distephanus major (Frenguelli), n. comb.} (Plate 7, Fig. 4)

Dictyocha boliviensis Frenguelli, 1940 (in part), p. 44, figs. 4b,c. Dictyocha boliviensis var. major Frenguelli, 1951, p. 277, figs. 3a-c. Cannopilus major (Frenguelli) Bukry and Foster, 1973 (in part), p. 826, pl. 1, figs. 4-7, (not pl. 7, fig. 4).

Remarks. Distephanus boliviensis specimens having divided apical rings, so-called cannopilean forms, differ from D. major in several aspects (Bukry, 1979b). The equant, short-spined, straight-sided, polyg- 
onal basal ring of $D$. major is distinctive in lower Pliocene Cores 28 to 30 at DSDP Hole 504.

\section{Distephanus mesophthalmus (Ehrenberg) Haecke}

Dictyocha mesophthalma Ehrenberg, 1844, p. 64, 80; Ehrenberg, 1854 , pl. 22, fig. 43.

Distephanus mesophthalmus (Ehrenberg) Haeckel, Bukry, in press c, pl. 5, fig. 3.

Remarks. The range for Distephanus mesophthalmus at DSDP sites has been extended upward considerably from its original range of upper middle to lower upper Miocene for DSDP Hole 21 (Dumitrică, 1973b). On the Pacific Coast, it occurs in the upper upper Miocene at DSDP Hole 471 (Bukry, in press d), and at DSDP Hole 504 it ranges to the upper upper Pliocene Dictyocha delicata Subzone of Core 18. Also, a re-examination of the abundant (16\%) "Distephamus crux", reported from upper Pliocene Core 11 core-catcher at nearby DSDP Hole 157 (Bukry and Foster, 1973) shows that those specimens possess apical pikes and now can be assigned to $D$. mesophthalmus. Although both of these newly cited occurrences are upper Pliocene, the DSDP Hole 157 sample contains Dictyocha ornata instead of $D$. delicata, and the two therefore are assigned to different subzones. The high (9-16\%) abundances of $D$. mesophthalmus is suggestive of close correlation potential beween DSDP Holes 157 and 504

\section{Distephanus polyactis (Ehrenberg) Deflandre}

(Plate 7, Fig. 5)

Dictyocha polyactis Ehrenberg, 1839, p. 129; Ehrenberg, 1854, pl. 22 , fig. 50 .

Distephanus polyactis (Ehrenberg) Deflandre, Bukry and Foster, 1973 , p. 828 , pl. 5 , figs. 6,7 .

Remarks. A single specimen was counted at DSDP Hole 504 in upper Miocene Core 53

\section{Distephanus quinquangellus Bukry and Foster (Plate 7, Fig. 6)}

Distephanus quinquangellus Bukry and Foster, 1973, p. 828, pl. 5, fig. 4 .

Remarks. At DSDP Hole 504, most specimens of Distephanus quinquangellus have apical pikes. This, together with smaller size and more-radial symmetry, helps to distinguish $D$. quinquangellus from Distephanus sp. A (Bukry, 1979a). Both pentagonal forms occur through the Pliocene and are most abundant in the upper Pliocene, but $D$. quinquangellus is missing from the Pleistocene. In Core 22, where $D$. quinquangellus is most abundant $(28 \%)$, some quadrangular variants could mimic Distephanus mesophthalmus, but these are recorded as $D$. sp. aff. $D$. mesophthalmus $(2 \%)$. Similarly, the $<1 \% D$. speculum $\mathrm{f}$. coronata from this sample could be a hexagonal variant. $D$. speculum f. coronata was noted sparsely and sporadically only in the Pliocene of DSDP Hole 504, where D. quinquangellus also is present. This implies that apical pikes are an ecophenotypic expression.

\section{Distephanus speculum bispicatus Bukry}

Distephanus speculum (Ehrenberg) Haeckel subsp. bispicatus Bukry, in press a, pl. 6, figs. 2-4.

Remarks. Like those of DSDP Hole 503A, the DSDP Hole 504 populations of Distephanus speculum bispicatus are most abundant (27\%) in the lower Pliocene. Only in Cores 29 and 43 does $D$. speculum bispicatus exceed $D$. speculum speculum. The only distinction of the $D$. speculum bispicatus distribution from that of $D$. speculum speculum at DSDP Hole 504 is that both Cores 29 and 43 coincide with transitional assemblages which fall between diagnostic subzone index ranges. Similar results are obtained at DSDP Hole 503A in Cores 16, 19 , and 24. Both range into the Pleistocene, but they are sparse and sporadic there. One of the specimens of $D$. sp. cf. $D$. frugalis in Core 49 has two apical pikes in the manner of $D$. speculum bispicatus. Therefore, this morphologic feature is not an effective taxonomic guide unless the additional characteristics of the type specimens are used.

\section{Distephanus speculum f. coronata Schulz} (Plate 7, Fig. 7)

Distephanus speculum f. coronata Schulz, 1928, p. 262, fig. 50.
Remarks. Distephanus speculum $\mathrm{f}$. coronata has a full cycle of six apical pikes, which distinguishes it from $D$. speculum.

\section{Distephanus speculum elongatus Bukry}

Distephanus speculum elongatus Bukry, 1975, p. 688, pl. 2, figs., 8,9; pl. 3, figs. 1-3.

\section{Distephanus speculum minutus Bachmann, emend. Bukry}

Dictyocha speculum f. minuta Bachmann in Ichikawa et al., 1967, p. 161 , pl. 7, figs. $12-15$.

Distephanus speculum minutus (Bachmann), emend. Bukry, in press d.

Remarks. This cool-water subspecies is recorded only in lower Pliocene Core 42, which has a low $T_{\mathrm{s}}$ value (57).

\section{Distephanus speculum speculum (Ehrenberg) Haeckel}

Dictyocha speculum Ehrenberg, 1839, p. 150; Ehrenberg, 1854, pl. 18, fig. 57 ; pl. 19 , fig. $41 ;$ pl. 21 , fig. $44 ;$ pl. 22 , fig. 47 .

Distephanus speculum speculum (Ehrenberg), Bukry, 1980b, p. 553, pl. 5, figs. 15,16; pl. 6, figs. 1-3.

\section{Distephanus speculum tenuis Bukry}

Distephanus speculum (Ehrenberg) Haeckel tenuis Bukry, in press a, pl. 6, figs. 5-11.

\section{Distephanus sp. A. Bukry}

(Plate 7, Fig. 8)

Distephanus sp. A Bukry, 1979a, p. 985, pl. 5, figs. 7-10.

Remarks. Larger size and less-perfect radial symmetry distinguish Distephanus sp. A (Bukry, 1979a) from D. quinquangellus. D. sp. A is most prominent in upper Pliocene or lowermost Pleistocene Cores 13 to 20 at DSDP Hole 504 .

\section{Genus MESOCENA Ehrenberg, 1843 Mesocena circulus (Ehrenberg) Ehrenberg} (Plate 8, Figs. 1,2)

Dictyocha (Mesocena) circulus Ehrenberg 1840, p. 208; Ehrenberg, 1854 , pl. 19 , fig. 44 as Mesocena. Mesocena circulus (Ehrenberg) Ehrenberg, 1844, p. 65. Mesocena circulus (Ehrenberg), Bukry, in press d.

Remarks. Mesocena circulus is a minor species in the Pliocene of DSDP 504. Abundance exceeds $3 \%$ only in Cores 41, 43, and 44, which are lower Pliocene. An unusual oblong variant occurs in Core 43. In Pleistocene Core 5, a spade-shaped specimen is tabulated as $M$. sp. cf. M. circulus (Plate 8, Fig. 3).

\section{Mesocena diodon nodosa Bukry}

(Plate 8, Fig. 4)

Mesocena diodon nodosa Bukry, 1978c (in part), p. 818, pl. 5, figs. 14,15 ; pl. 6 , figs. $1-3$ (not figs. 4,5).

Remarks. The sparse Mesocena diodon nodosa in Cores 51 to 53 are in place, with typically associated upper upper Miocene species. The single occurrence above this level is a displaced specimen.

Because the basionym $M$. crenulata was considered a nomen nudum (Loeblich et al., 1968), the formation $M$. diodon nodosa was proposed (Bukry, 1978c) to classify obviously noded specimens related to smooth $M$. diodon s. str.

\section{Mesocena quadrangula Ehrenberg ex Haeckel}

(Plate 8, Figs. 5-10; Plate 9, Figs. 1-10)

Mesocena quadrangula Ehrenberg ex Haeckel, 1887, p. 1556; Lemmermann, 1901, pl. 10, figs. 5-7; fide Loeblich et al., 1968, p. 57. Mesocena quadrangula Ehrenberg ex Haeckel, 1887, Bukry, 1979b, p. 574 , pl. 5, figs. 5,6 .

Remarks. Within the Pleistocene acme of Mesocena quadrangula in Cores 6 to 10 there is a slight change to smaller size up-section. The contrast in size between larger specimens in Cores 9 and 10 and smaller in Core 8 is also augmented by the presence of four major peripheral pikes on the rings of many $(>10 \%)$ of the specimens from Core 8 . This morphologic feature is not present in the Core 9 or 10 assem- 
blages. The upper Miocene $M$. quadrangula population of Cores 52 and 53 contains elongated and distorted forms with sides bowed in or out. Upper Miocene distorted forms also are illustrated from nearby DSDP Hole 157 (Bukry and Foster, 1973), and may provide a local correlation guide.

One specimen from Core 50 is tabulated as transennoid because it has a short bar in one of the ring corners; it could be a mesocenoid variant of a Dictyocha species.

\section{Mesocena triodon Bukry}

Mesocena triodon Bukry, 1973, p. 860, pl. 2, fig. 11.

\section{Genus OCTACTIS Schiller, 1926 \\ Octactis pulchra Schiller \\ (Plate 7, Fig. 10)}

Octactis pulchra Schiller, 1926, p. 67, fig. c.

Remarks. Octactis pulchra is limited to Cores 1 to 12 of DSDP Hole 504, which contain Pleistocene assemblages. Slightly higher abundances in the Dictyocha aculeata Zone may suggest increased mixing and upwelling (see Donegan and Schrader, 1981, p. 151).

\section{ACKNOWLEDGMENTS}

The shipboard diatom biostratigraphy and ages for DSDP Legs 68 and 69 by Constance Sancetta, Lamont-Doherty Geological Observatory, greatly assisted the comparison between silicoflagellate paleotemperature values at DSDP Holes 503A and 504. I thank John A. Barron and Gerta Keller, U.S. Geological Survey, for constructive criticism of the manuscript and for recommendations on curve representation. The manuscript and tables were ably typed and proofed by Dorothy Blackstock, U.S. Geological Survey. I thank Roy Couzin, U.S. Geological Survey, for final drafting of the paleotemperature curves.

\section{REFERENCES}

Barron, J. A., 1980. Upper Pliocene and Quaternary diatom biostratigraphy of Deep Sea Drilling Project Leg 54, tropical eastern Pacific. In Hekinian, R., Rosendahl, B. R., et al., Init. Repts. DSDP, 54: Washington (U.S. Govt. Printing Office), 455-485.

Bukry, D., 1973. Coccolith and silicoflagellate stratigraphy, Deep Sea Drilling Project Leg 18, eastern North Pacific. In Kulm, L. D., von Huene, R., et al., Init. Repts. DSDP, 18: Washington (U.S. Govt. Printing Office), 817-831.

1975. Coccolith and silicoflagellate stratigraphy, northwestern Pacific Ocean, Deep Sea Drilling Project Leg 32. In Larson, R. L., Moberly, R., et al., Init. Repts. DSDP, 32: Washington (U.S. Govt. Printing Office), 677-701.

1976. Silicoflagellate and coccolith stratigraphy, southeastern Pacific Ocean, Deep Sea Drilling Project Leg 34. In Yeats, R. S., Hart, S. R., et al., Init. Repts. DSDP, 34: Washington (U.S. Govt. Printing Office), 715-735.

1977. Coccolith and silicoflagellate stratigraphy, central North Atlantic Ocean, Deep Sea Drilling Project Leg 37. In Aumento, F., Melson, W. G., et al., Init. Repts. DSDP, 37: Washington (U.S. Govt. Printing Office), 917-927.

1978a. Cenozoic silicoflagellate and coccolith stratigraphy, southeastern Atlantic Ocean, Deep Sea Drilling Project Leg 40. In Bolli, H. M., Ryan, W. B. F., et al., Init. Repts. DSDP, 40: Washington (U.S. Govt. Printing Office), 635-649.

1978b. Cenozoic coccolith and silicoflagellate stratigraphy, offshore northwest Africa, Deep Sea Drilling Project Leg 41. In Lancelot, Y., Seibold, E., et al., Init. Repts. DSDP, 41: Washington (U.S. Govt. Printing Office), 689-707.

1978c. Cenozoic coccolith, silicoflagellate, and diatom stratigraphy, Deep Sea Drilling Project Leg 44. In Benson, W. E., Sheridan, R. E, et al., Init. Repts. DSDP, 44: Washington (U.S. Govt. Printing Office), 807-863.

1979 a. Comments on opal phytoliths and stratigraphy of Neogene silicoflagellates and coccoliths at Deep Sea Drilling Project Site 397 off northwest Africa. In Luyendyk, B. P, Cann, J. R., et al., Init. Repts. DSDP, 49: Washington (U.S. Govt. Printing Office), $977-1009$

1979b. Coccolith and silicoflagellate stratigraphy, northern Mid-Atlantic Ridge and Reykjanes Ridge, Deep Sea Drilling Proj- ect Leg 49. In Luyendyk, B. P., Cann, J. R., et al., Init. Repts. $D S D P$, 49: Washington (U.S. Govt. Printing Office), 551-581. 1980a. Miocene Corbisema triacantha Zone phytoplankton from Deep Sea Drilling Project Sites 415 and 416, off northwest Africa. In Lancelot, Y., Winterer, E. L., et al., Init. Repts. DSDP, 50: Washington (U.S. Govt. Printing Office), 507-523.

$1980 \mathrm{~b}$. Silicoflagellate biostratigraphy and paleoecology in the eastern equatorial Pacific, Deep Sea Drilling Project Leg 54. In Hekinian, R., Rosendahl, B. R., et al., Init. Repts. DSDP, 54: Washington (U.S. Govt. Printing Office), 545-573. in press a. Neogene silicoflagellates of the eastern equatorial Pacific, Deep Sea Drilling Project Hole 503A. In Prell, W. L., Gardner, J. V., et al., Init. Repts. DSDP, 68: Washington (U.S. Govt. Printing Office).

in press b. Synthesis of silicoflagellate stratigraphy for Maestrichtian to Quaternary marine sediment. Soc. Econ. Paleontol. Mineral. Spec. Publ., 32. in press c. Cenozoic silicoflagellates from offshore Guatemala, Deep Sea Drilling Project Site 495. In von Huene, R., Auboin, J., et al., Init. Repts. DSDP, 67: Washington (U.S. Govt. Printing Office). in press d. Silicoflagellate stratigraphy of offshore California and Baja California, Deep Sea Drilling Project Leg 63. In Yeats, R. S., Haq, B. U., et al., Init. Repts. DSDP, 63: Washington (U.S. Govt. Printing Office).

Bukry, D., and Foster, J. H., 1973. Silicoflagellate and diatom stratigraphy, Leg 16, Deep Sea Drilling Project. In van Andel, Tj. H., Heath, G. R., et al., Init. Repts. DSDP, 16: Washington (U.S. Govt. Printing Office), 815-871.

Burckle, L. H, 1977. Pliocene and Pleistocene diatom datum levels for the equatorial Pacific. Quat. Res., 7:330-340.

Ciesielski, P. F., 1975. Biostratigraphy and paleoecology of Neogene and Oligocene silicoflagellates from cores recovered during Antarctic Leg 28, Deep Sea Drilling Project. In Hayes, D. E., Frakes, L. A., et al., Init. Repts. DSDP, 28: Washington (U.S. Govt. Printing Office), 625-691.

Donegan, D., and Schrader, H., 1981. Modern analogues of the Miocene diatomaceous Monterey shale of California: Evidence from sedimentologic and micropaleontologic study. In Garrison, R. E., et al. (Eds.) The Monterey Formation and Related Siliceous Rocks of California. Soc. Econ. Paleontol. Mineral. Pacific Sec., Spec. Publ., pp. 149-157.

Dumitrica, P., 1973a. Miocene and Quaternary silicoflagellates in sediments from the Mediterranean Sea. In Ryan, W. B. F., Hsü, K. J., et al., Init. Repts. DSDP, 13: Washington (U.S. Govt. Printing Office), 902-933.

1973b. Paleocene, late Oligocene and post-Oligocene silicoflagellates in southwestern Pacific sediments cored on DSDP Leg 21. In Burns, R. E., Andrews, J. E., et al., Init. Repts. DSDP, 21: Washington (U.S. Govt. Printing Office), 837-883.

1978. Badenian silicoflagellates from Central Paratethys. In Brestenská, E. (Ed.), Chronostratigraphie und Neostratotypen, Miozän der Zantralen Paratethys (Vol. 6): Bratislava (Veda), 207-230.

Ehrenberg, C. G., 1839. Über die Bildung der Kreidefelsen und des Kreidemergels durch unsichtbare Organismen. K. Preuss. Akad. Wiss. Berlin Ber., Jahrg. 1838:59-148.

1840. 274 Blätter von ihm selbst augeführter Zeichnungen von ebenso vielen Arten. $K$. Preuss. Akad. Wiss. Berlin Ber., Jahrg. 1840:197-129 (Nov.).

1844. Mittheilung über zwei neue Lager von Gebirgsmassen aus Infusorien als Meeres-Absatz in Nord-Amerika und eine Vergleichung derselben mit den organischen Kreide-Gebilden in Europa und Afrika. K. Preuss. Akad. Wiss. Berlin. Ber., Jahrg. 1844: 57-97.

1854. Mikrogeologie: Leipzig (Leopold Voss).

Frenguelli, J., 1940. Consideraciones sobre los sílicoflagelados fósiles. Mus. La Plata Rev., Paleontol., 2:37-112.

1951. Silicoflagelados del Tripoli de Mejillones (Chile). Physis [Buenos Aires], 20:272-284.

Haeckel, E. H. P. A., 1887. Cannorrhaphida. Challenger Rept., 18: 1546-1569.

Ichikawa, W., Shimizu, I., and Bachmann, A., 1967. Fossil silicoflagellates and their associated uncertain forms in Iida Diatomite, 
Noto Peninsula, Central Japan. Kanazawa Univ. Sci. Repts., 12: 143-172.

Keigwin, L. D., Jr., 1979. Late Cenozoic stable isotope stratigraphy and paleoceanography of DSDP sites from the eastern equatorial and central North Pacific Ocean. Earth Planet. Sci. Lett., 45: 361-382.

Lemmermann, E., 1901. Silicoflagellatae. Deutsche. Bot. Gesell. Ber., 19:247-271.

Locker, S., 1975. Dictyocha varia sp. n., eine miozane Silicoflagellaten-Art mit kompliziertem Variationsmodus. Zeitschr. Geol. Wiss., Berlin, 3:99-103.

Loeblich, A. R., III. Loeblich, L. A., Tappan, H., and Loeblich, A. R., Jr. 1968. Annotated index of fossil and recent silicoflagellates and ebridians with descriptions and illustrations of validly proposed taxa. Geol. Soc. Am. Mem., 106.

Martini, E., 1971. Neogene silicoflagellates from the equatorial $\mathrm{Pa}-$ cific. In Winterer, E. L., Riedel, W. R., et al., Init. Repts. DSDP, 7, Pt. 2: Washington (U.S. Govt. Printing Office), 1695-1708.
Morlotti, E., and Rio, D., 1978 [1980]. Silicoflagellates, ebridians and actiniscids from Le Castella Section (Calabria, Southern Italy). Ateneo Parmense, Acta Nat., 14:95-118.

Poelchau, H. S., 1976. Distribution of Holocene silicoflagellates in North Pacific sediments. Micropaleontol., 22:164-193.

Schiller, J., 1926. Die planktontischen Vegetation des adriatischen Meers.: B. Chrysomonadina, Heterokontae, Cryptomonadina, Eugleninae, Volvocales. 1. Systematischer Teil. Arch. Protistenk., 53: 59-123.

Schulz, P., 1928. Beiträge zur Kenntnis fossiler und rezenter Silicoflagellaten. Bot. Archiv, 21:225-292.

Stradner, H., and Bachmann, A., 1978. Late Pliocene and early Pleistocene silicoflagellates and ebridians from DSDP Site 378 in the Aegean Basin, north of Crete. In Hsü, K. J., Montadert, L., et al., Init. Repts. DSDP, 42, Pt. 1: Washington (U.S. Govt. Printing Office), 805-815. 


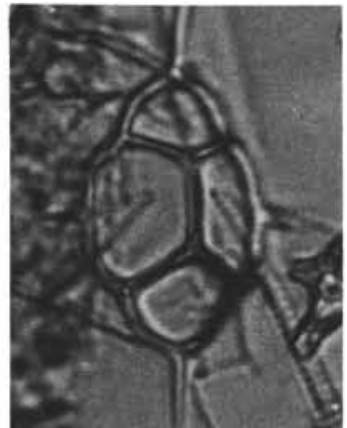

1

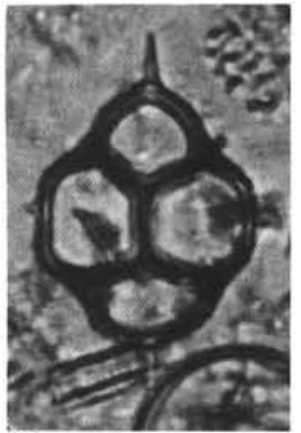

2

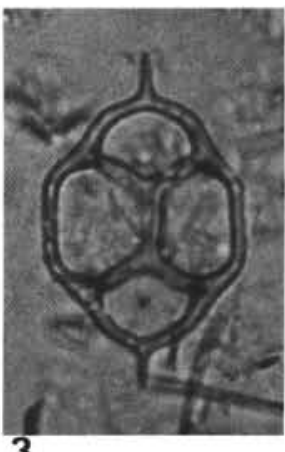

3

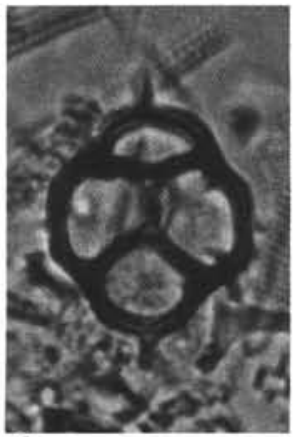

4

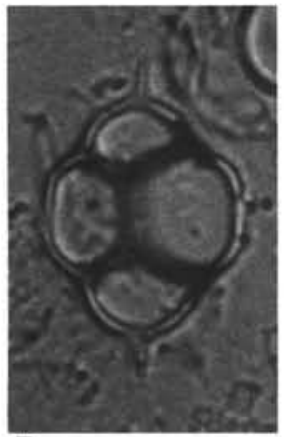

5

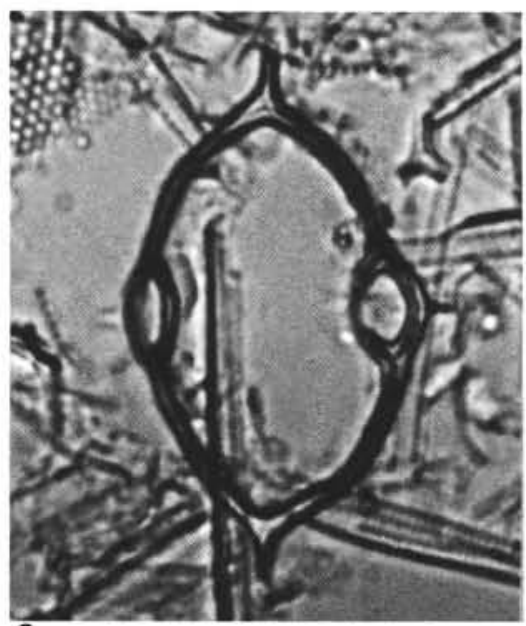

6

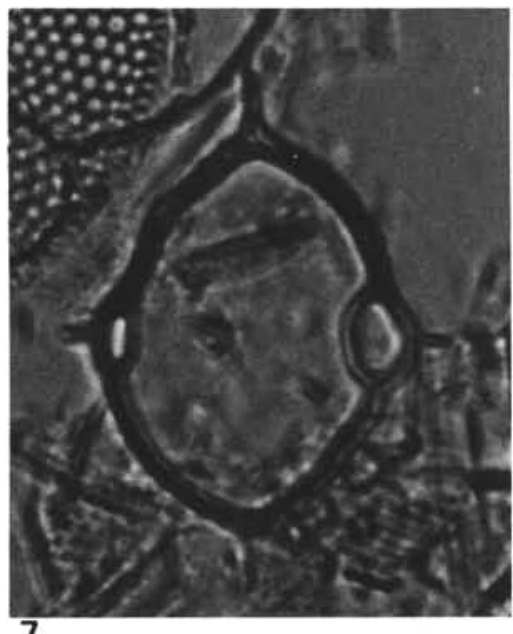

7
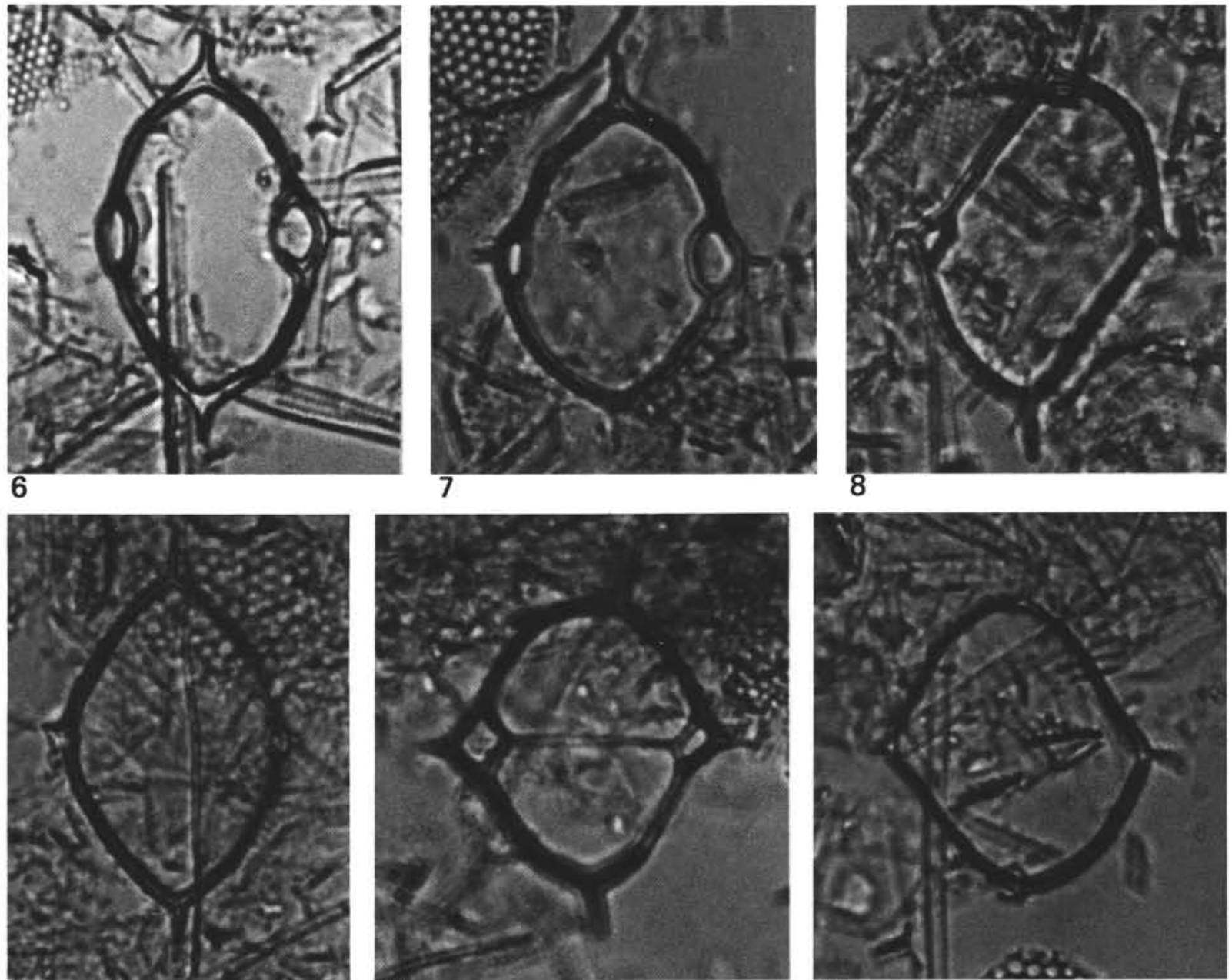

9

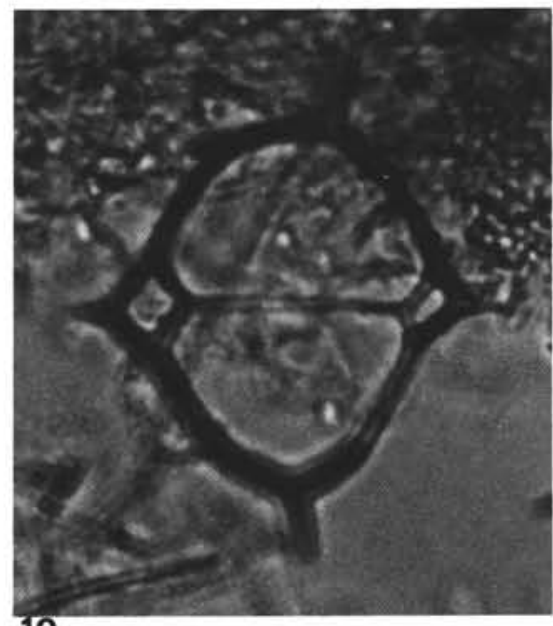

10

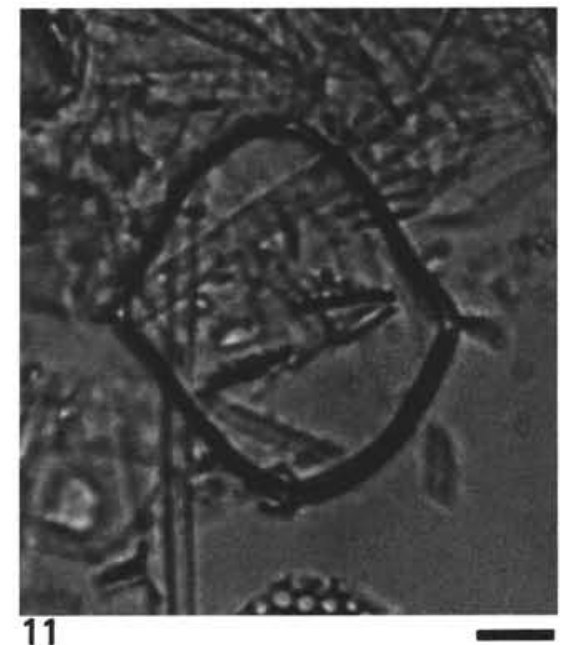

11

Plate 1. Silicoflagellates from DSDP Hole 504. Magnification $800 \times$; scale bar equals $10 \mu \mathrm{m}$. 1. Dictyocha sp. aff. D. aegea Stradner and Bachmann, Sample 504-26-1, 42-43 cm. 2-5. Dictyocha angulata Bukry. (2) Normal, Sample 504-36,CC. (3) Minor-axis spines missing, Sample 504-36,CC. (4,5) Sample 504-42-2, 42-43 cm. 6-9. Dictyocha brevispina (Lemmermann) (no bar); all from Sample 504-45-1, 42-43 cm. (6,7) Typical specimens. (8) Distorted. (9) Nearly mesocenoid with one small portal. 10,11. Dictyocha brevispina (Lemmermann); apically thinned and mesocenoid specimens with the same ring form from Sample 504-45-1, 42-43 cm. 


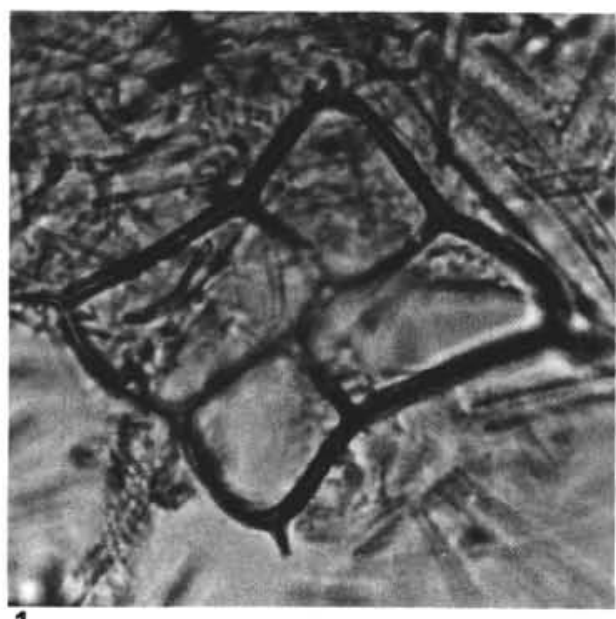

1

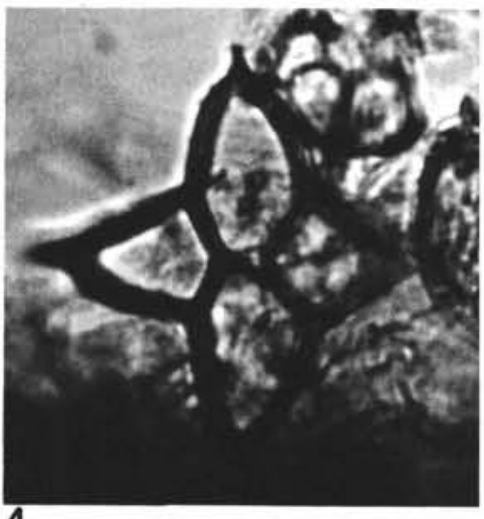

4

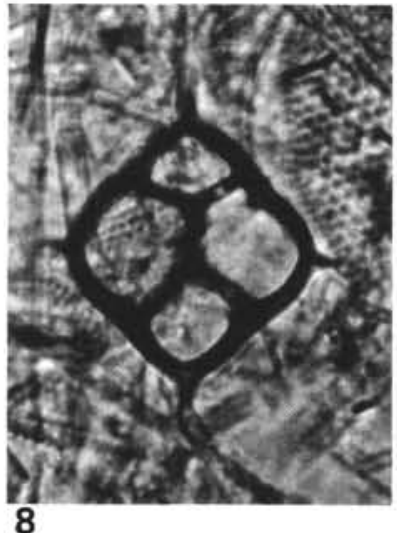

5

9

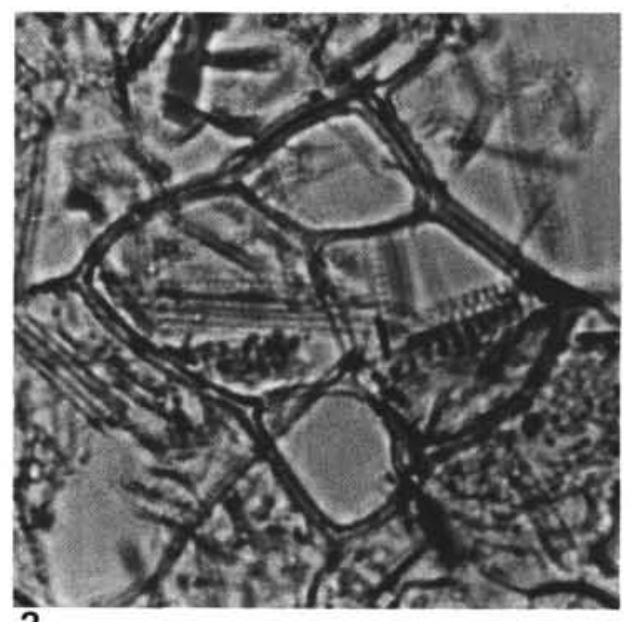

2
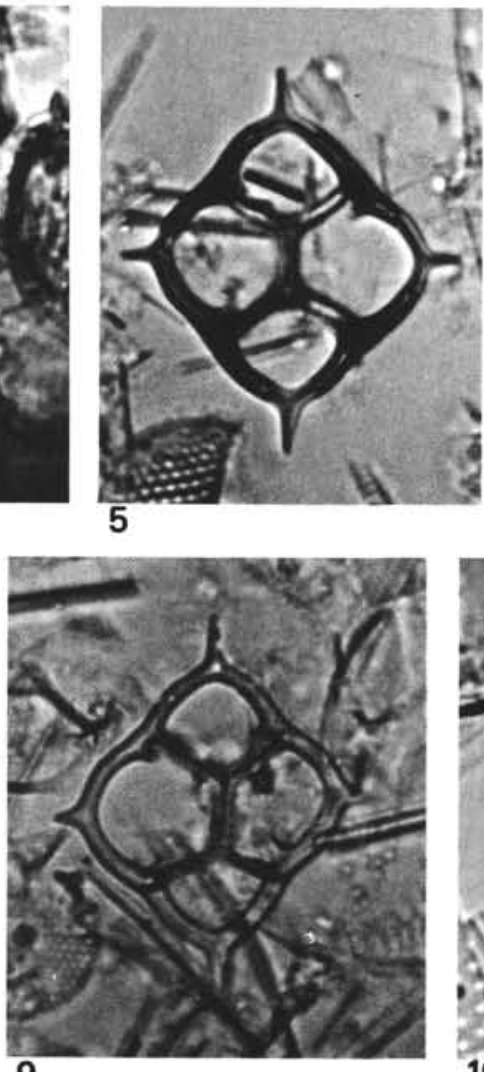

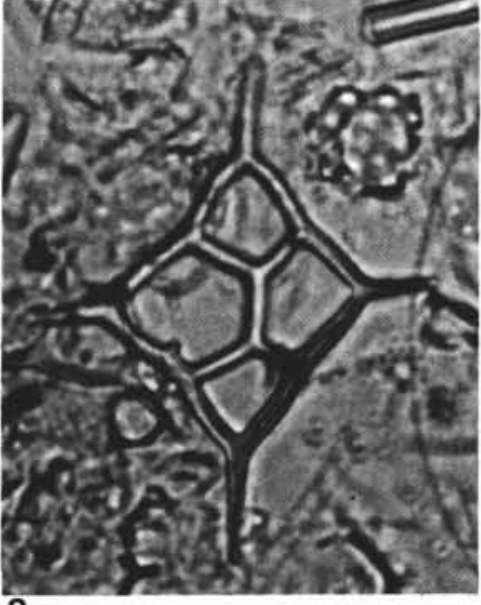

3
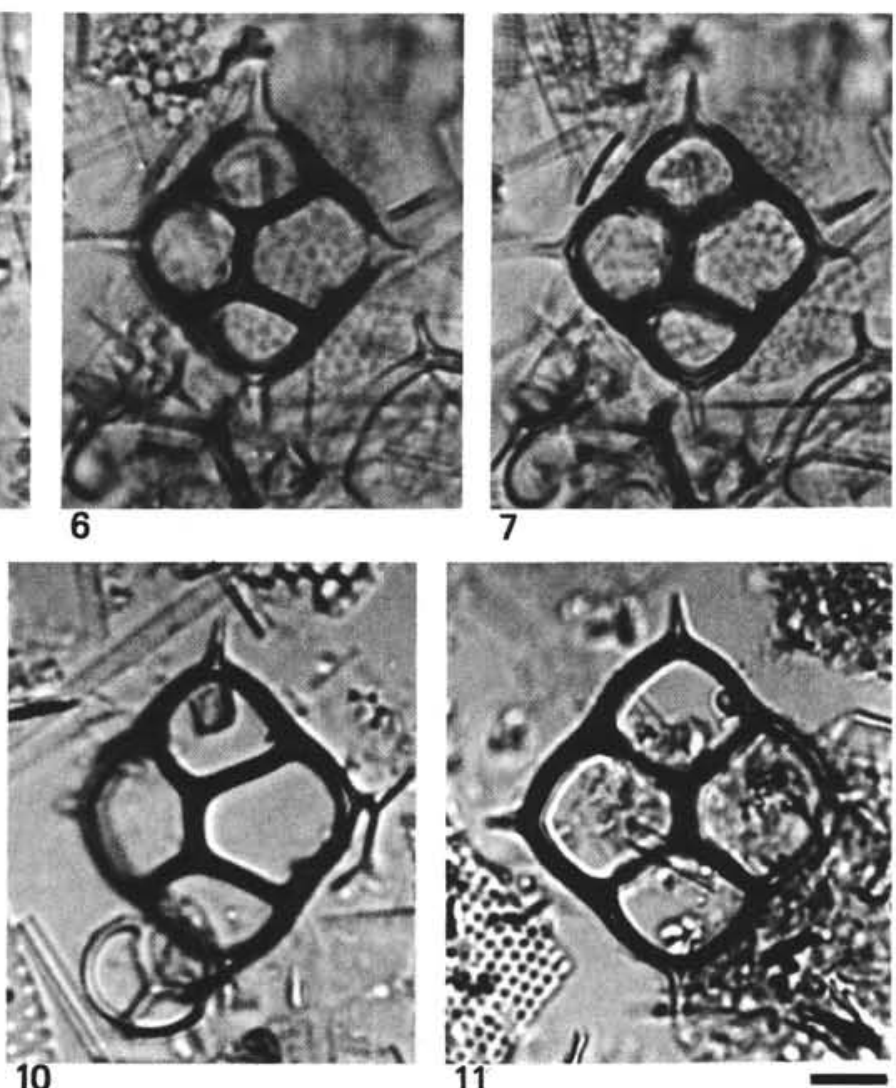

Plate 2. Silicoflagellates from DSDP Hole 504. Scale bar equals $10 \mu \mathrm{m} . \quad 1,2$. Dictyocha brevispina (Lemmermann) (giant asymmetric). (1) Sample 504-50-1, 42-43 cm. (2) Sample 504-49-1, 42-43 cm. 3. Dictyocha concavata Dumitrică, Sample 504-42-1, 42-43 cm. 4. Dictyocha calida ampliata Bukry (extremely elongate), Sample 504-5-2, 42-43 cm. 5-11. Dictyocha concinna Bukry, n. sp.; all specimens from Sample 504-24-1, 42-43 cm, except Figures 10 and 11 from Sample 503A-13-2, 21-22 cm. (5) Holotype, USNM 321351. (6,7) USNM 321352, high and low focus. (8) USNM 321353. (9) USNM 321354. (10) USNM 321355. (11) USNM 321356. 

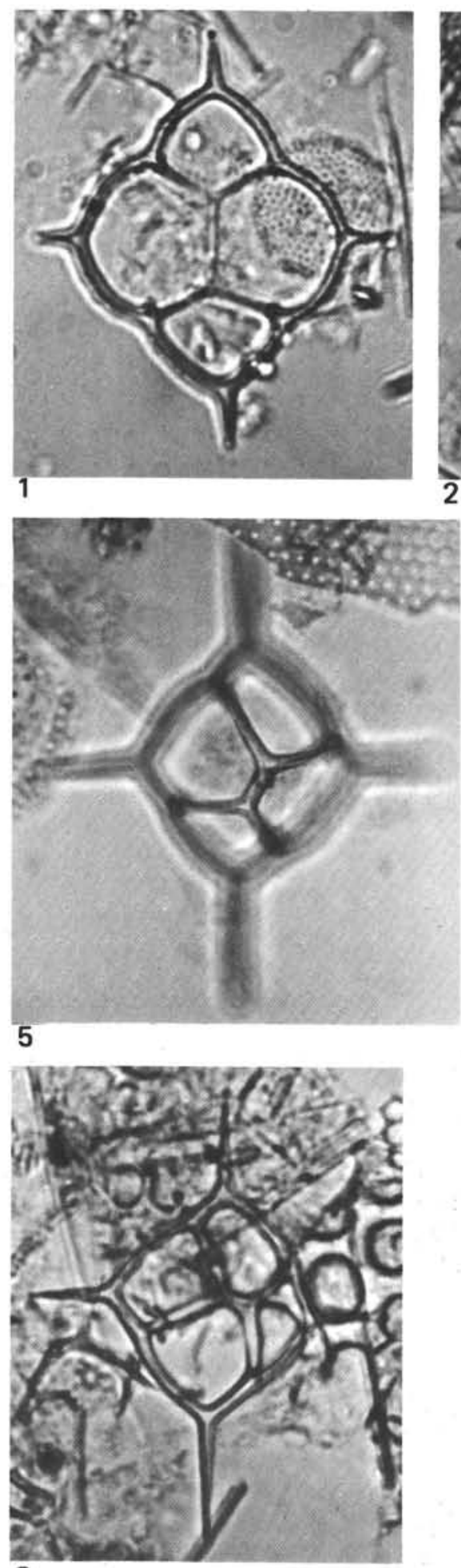

8

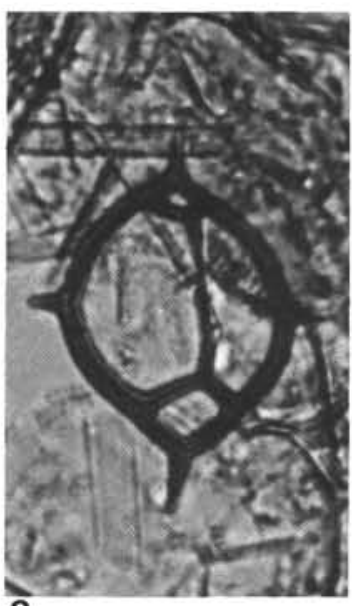

2

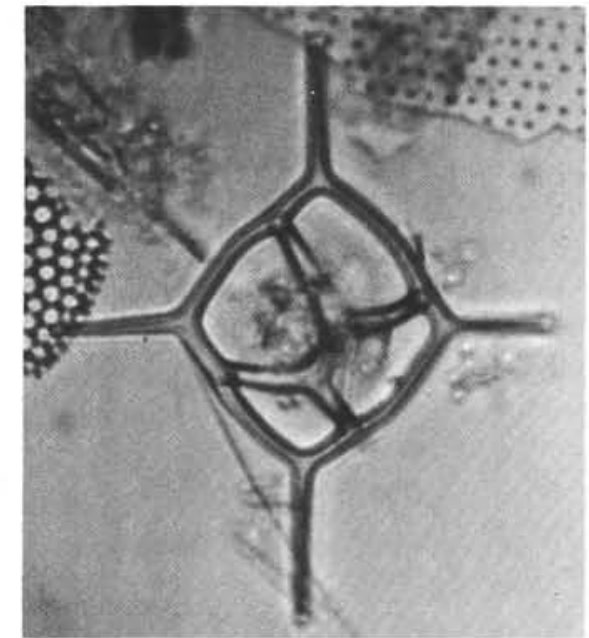

6

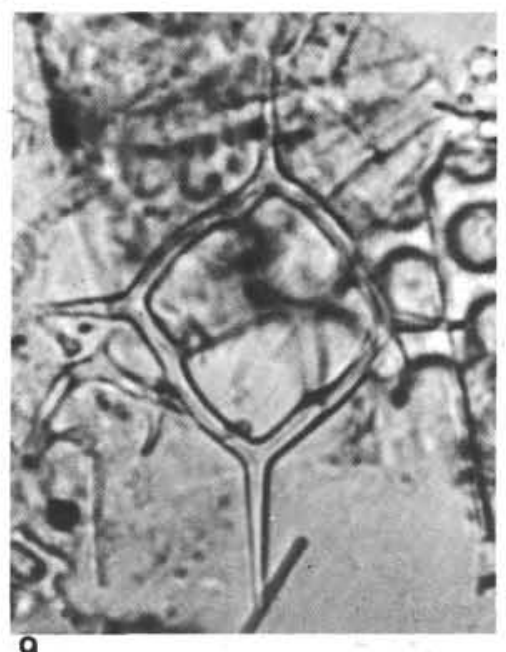

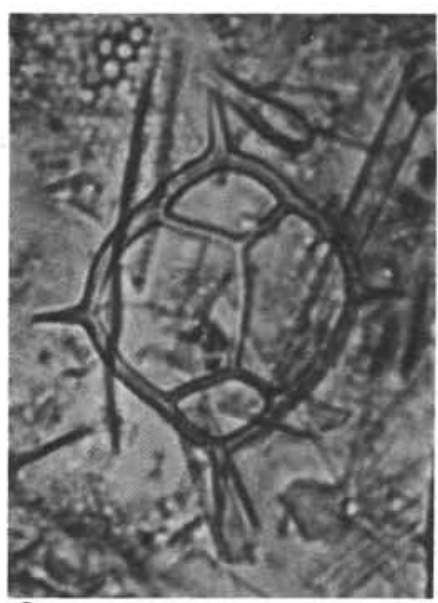

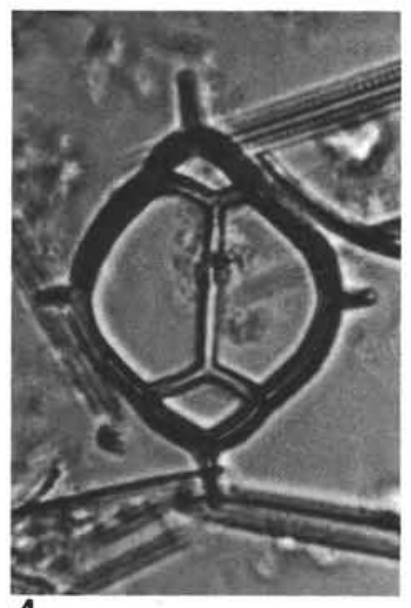

4
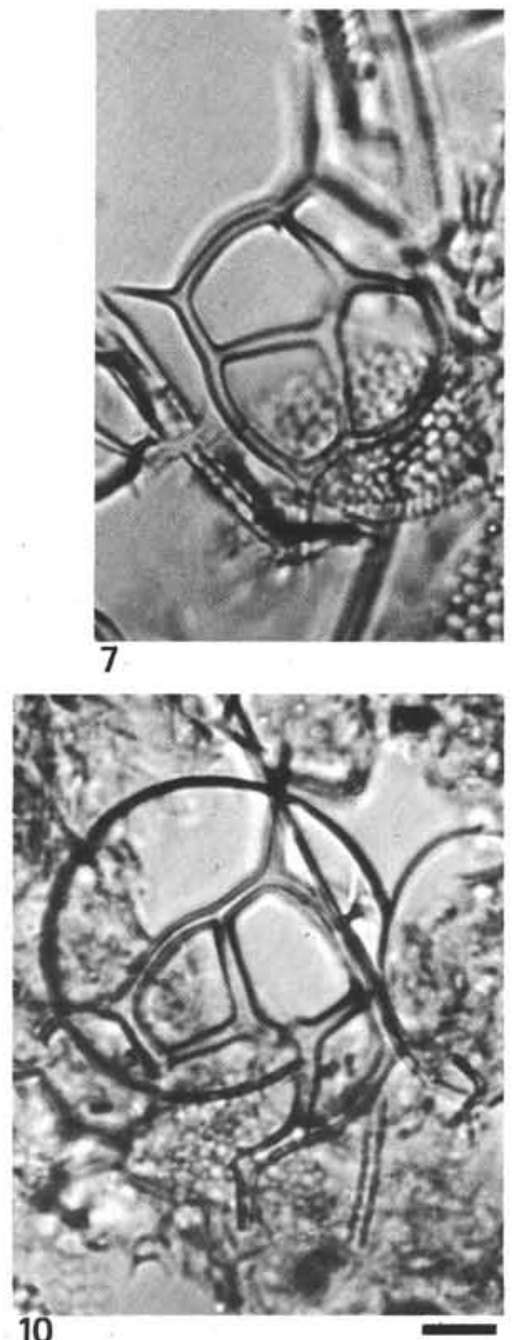

Plate 3. Silicoflagellates from DSDP Hole 504. Scale bar equals $10 \mu \mathrm{m}$. 1. Dictyocha delicata (Bukry), Sample 504-13-3, 42-43 cm. 2-4. Dictyocha delicata var. bisecta Bukry. (2,3) Normal, Sample 504-30-1, 42-43 cm. (4) Thick bar, Sample 504-31-1, 42-43 cm. 5-10. Dictyocha helix Bukry, n. sp. (5,6) Holotype, USNM 321357, Sample 504-19-2, 42-43 cm, high and low focus. (7) USNM 321358, Sample 504-19-2, 42-43 cm. $(8,9)$ USNM 321359, Sample 504-19-2, $42-43 \mathrm{~cm}$. (10) USNM 321360, Sample 504-18-3, $42-43 \mathrm{~cm}$. 

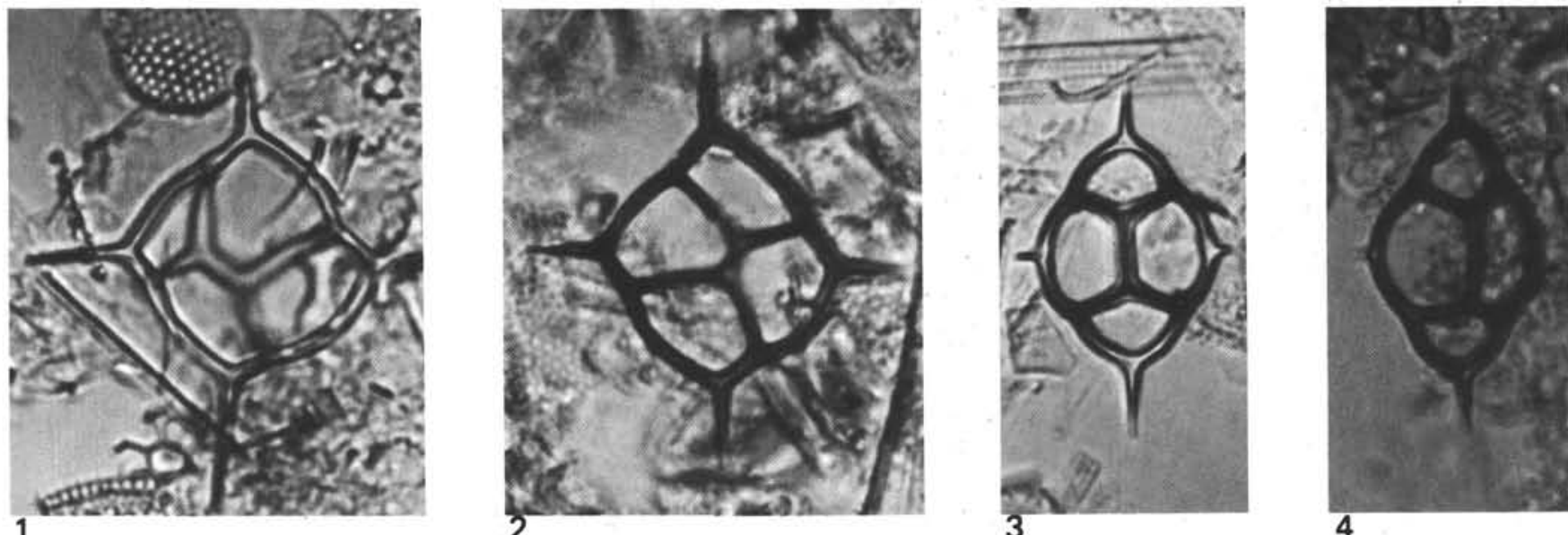
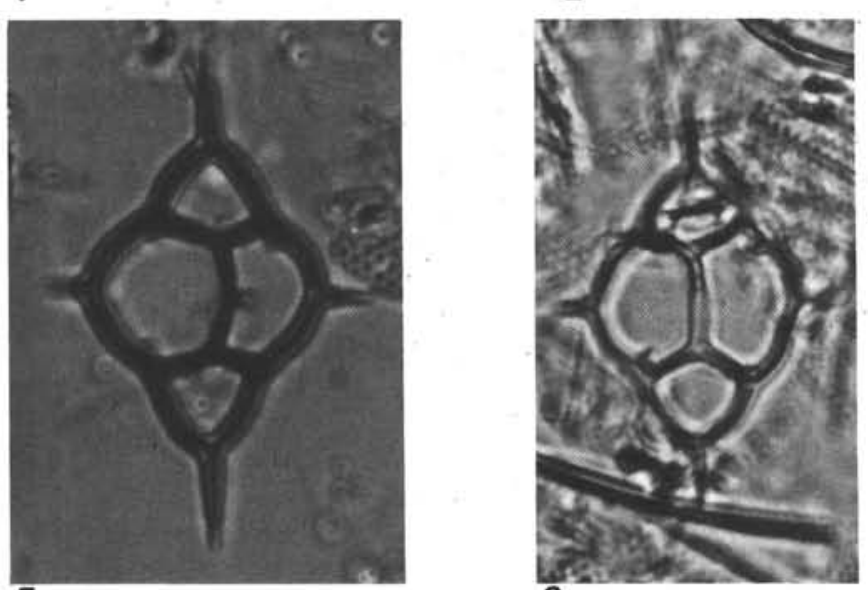

6

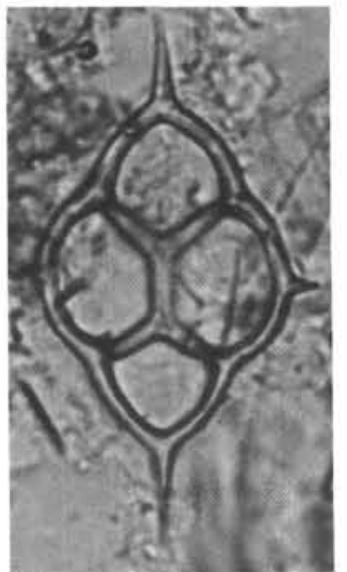

9

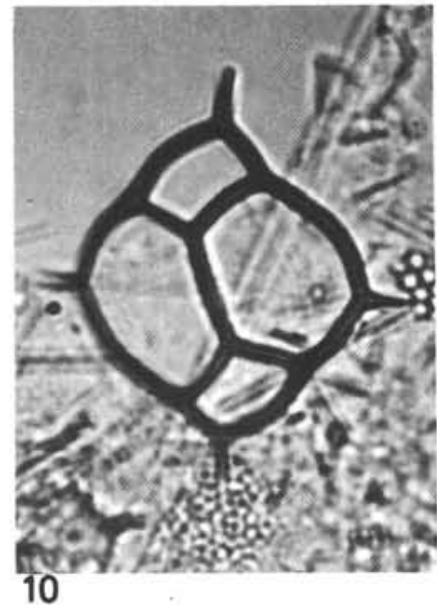

10

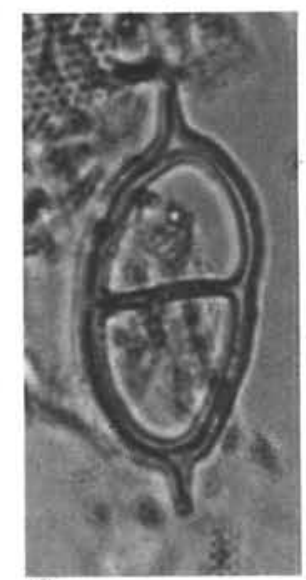

7

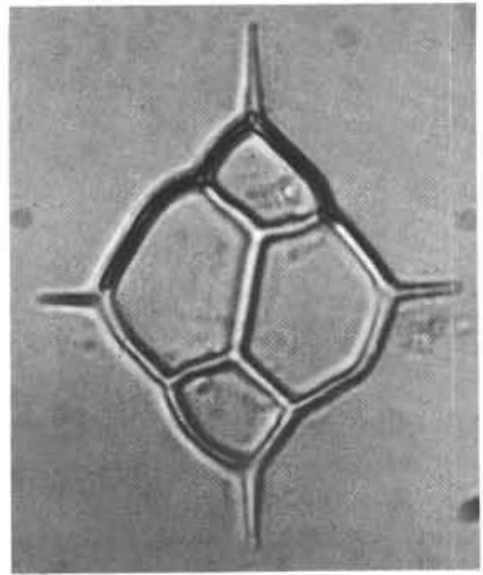

11

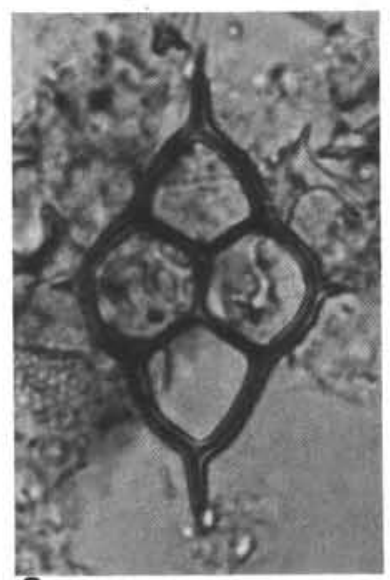

8

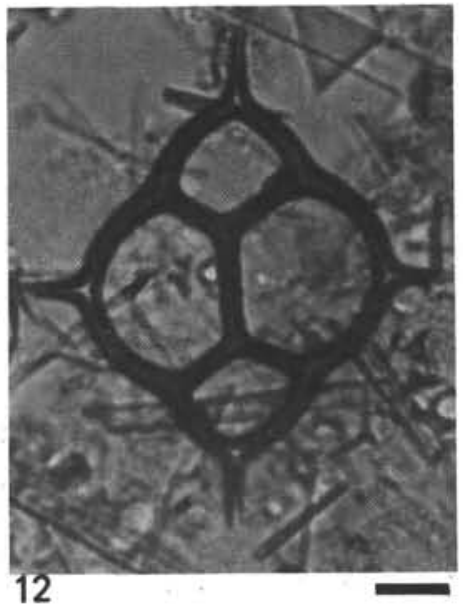

Plate 4. Silicoflagellates from DSDP Hole 504. Scale bar equals $10 \mu \mathrm{m} . \quad 1,2$. Dictyocha helix Bukry, n. sp. (1) USNM 321361, Sample 504-19-2, $42-43 \mathrm{~cm}$. (2) USNM 321362, Sample 504-20-2, 42-43 cm. 3,4. Dictyocha longa Bukry. (3) Sample 504-25-3, 42-43 cm. (4) Sample 504-32-1, $42-43 \mathrm{~cm}$. 5,6. Dictyocha longa var. paxilla Bukry. (5) Sample 504-33-1, 42-43 cm. (6) Sample 504-41-1, 42-43 cm. 7. Dictyocha neonautica var. cocosensis Bukry, Sample 504-52-1, 42-43 cm. 8,9. Dictyocha perfecta Bukry, Sample 504-37-1, 42-43 cm. 10,11. Dictyocha perlaevis flexatella Bukry. (10) Unusually sinuous, Sample 504-23-1, 42-43 cm. (11) Sample 504-26-1, 42-43 cm. 12. Dictyocha perlaevis perlaevis Frenguelli, Sample 504-30-1, 42-43 cm. 


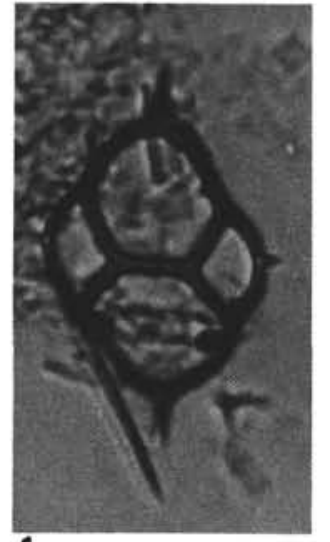

1

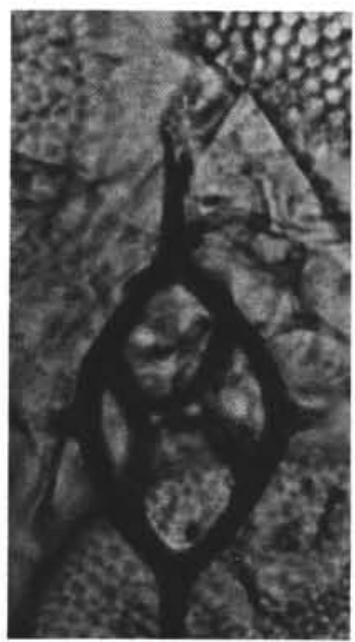

5

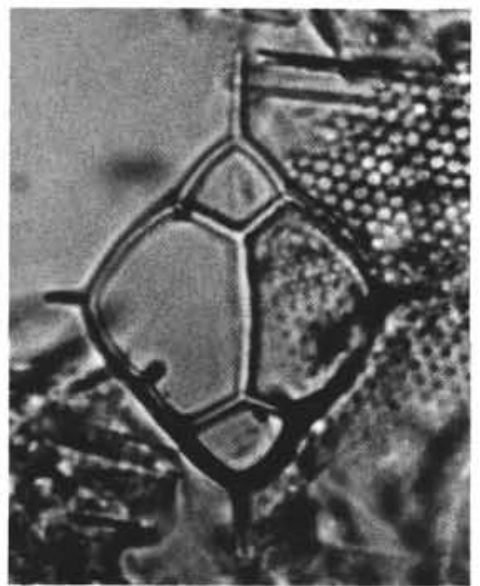

9

6
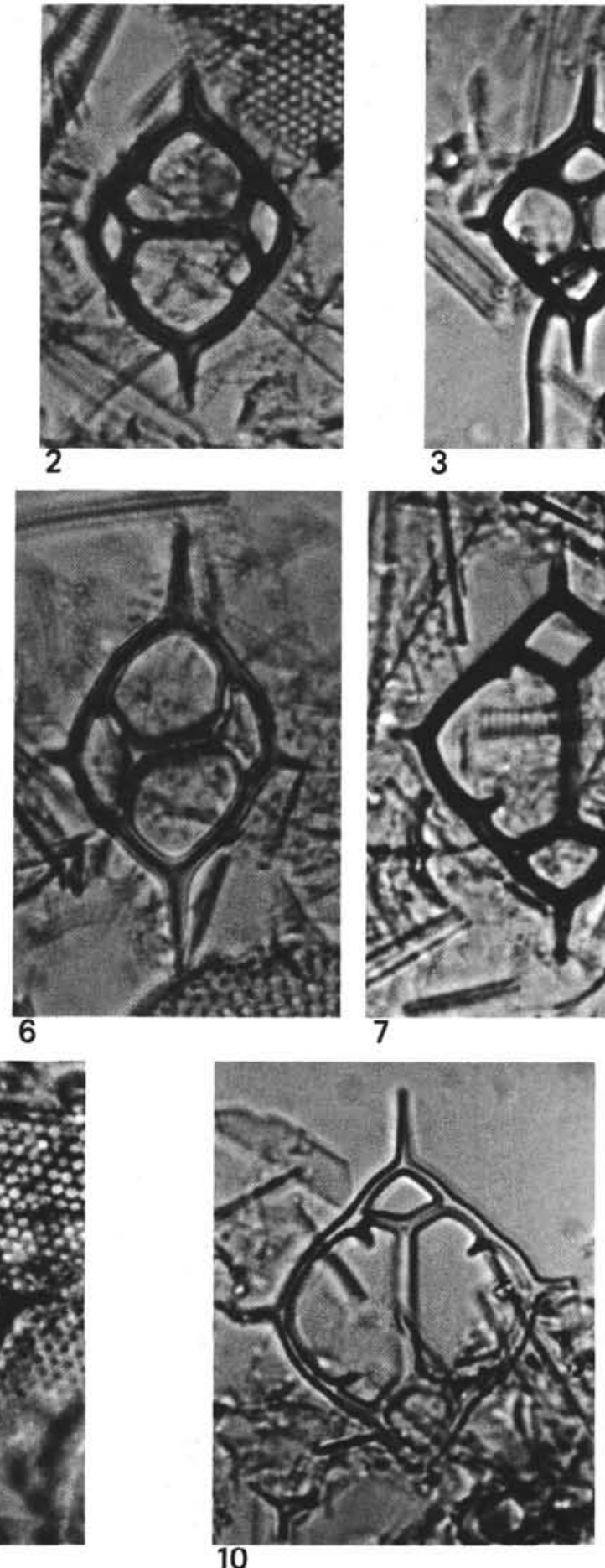

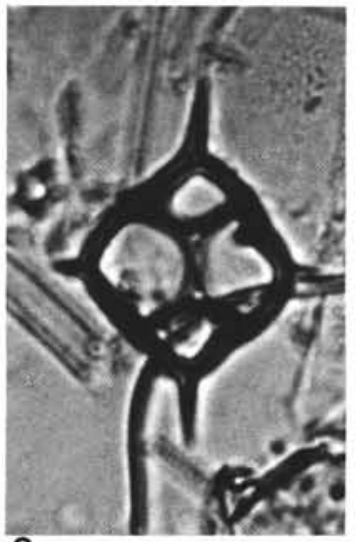

3

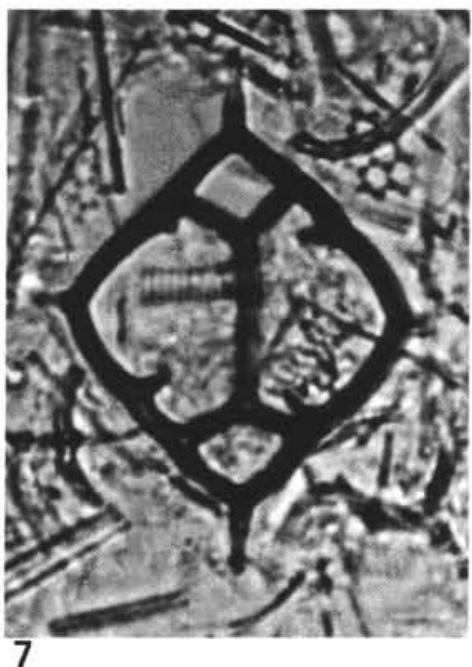

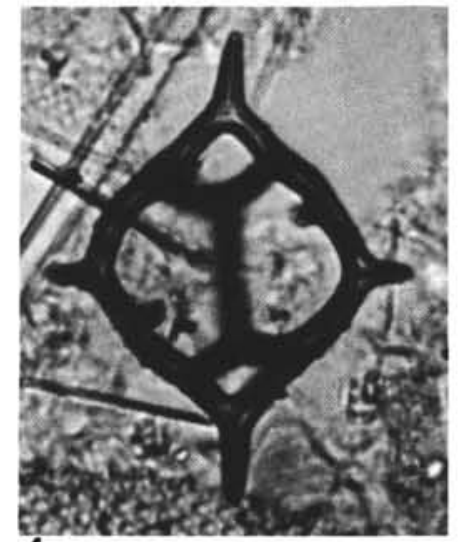

4

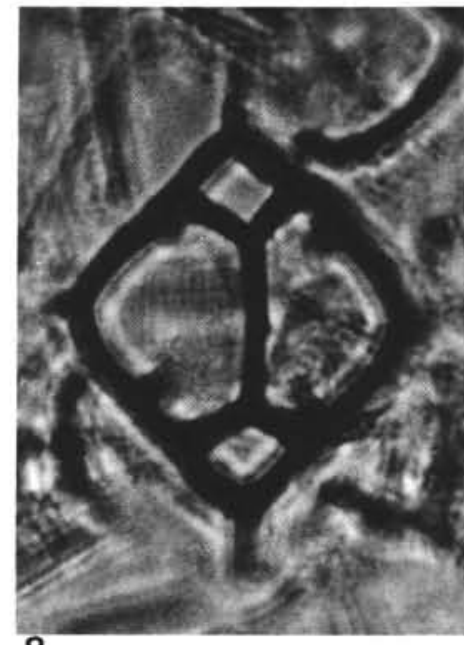

8

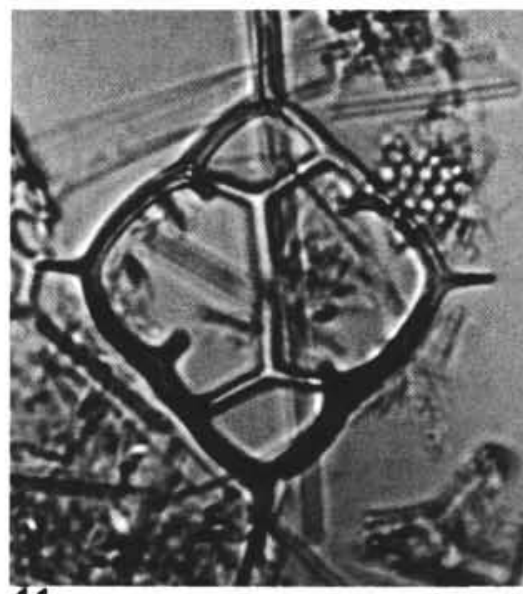

11

Plate 5. Silicoflagellates from DSDP Hole 504. Scale bar equals $10 \mu \mathrm{m} . \quad 1$. Dictyocha pulchella Bukry, Sample 504-52-1, 42-43 cm. 2. Dictyocha sp. aff. D. pulchella Bukry (angular), Sample 504-52-1, 42-43 cm. 3. Dictyocha sp. aff. D. stapedia aspinosa Bukry, Sample 504-23-1, $42-43 \mathrm{~cm}$. 4. Dictyocha sp. aff. D. subaculeata (Bukry), Sample 504-11-2, 42-43 cm. 5,6. Dictyocha sp. aff. D. subclinata Bukry, Sample 504-53-1, 26-27 cm. 7-11. Dictyocha tamarae Bukry, n. sp. (7,8) Holotype, USNM 321363, Sample 504-17-1, 42-43 cm, low and high focus. (9) USNM 321364, Sample 504-16-2, 42-43 cm. (10) USNM 321365, Sample 504-16-2, $42-43 \mathrm{~cm}$. (11) USNM 321366, Sample 504-16-2, 42-43 $\mathrm{cm}$. 


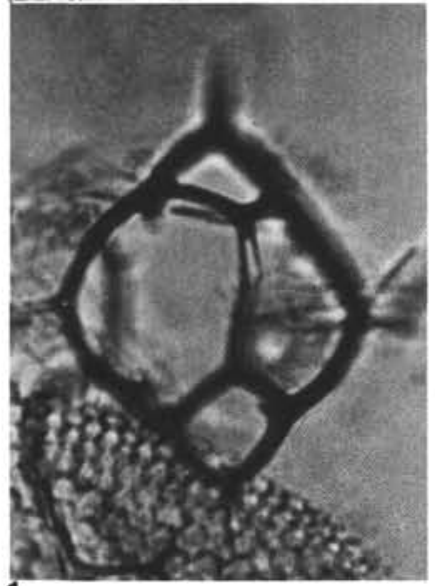
1

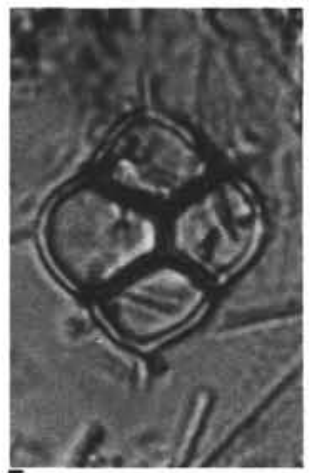

5

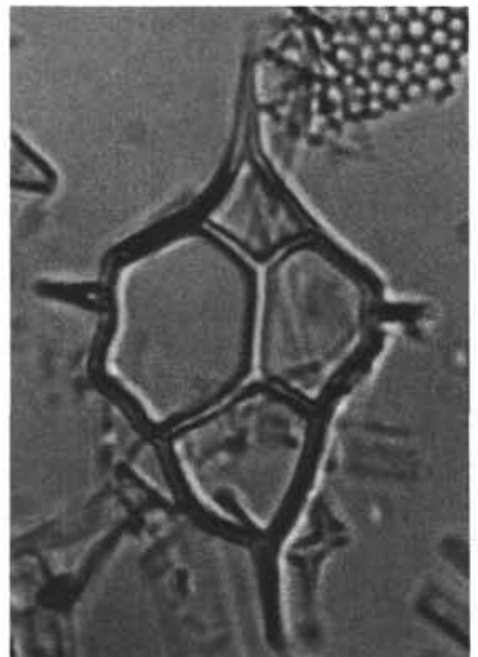

10

6

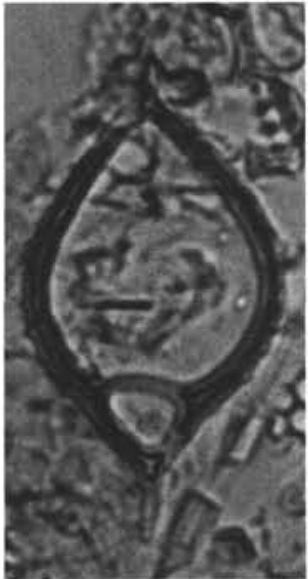

2
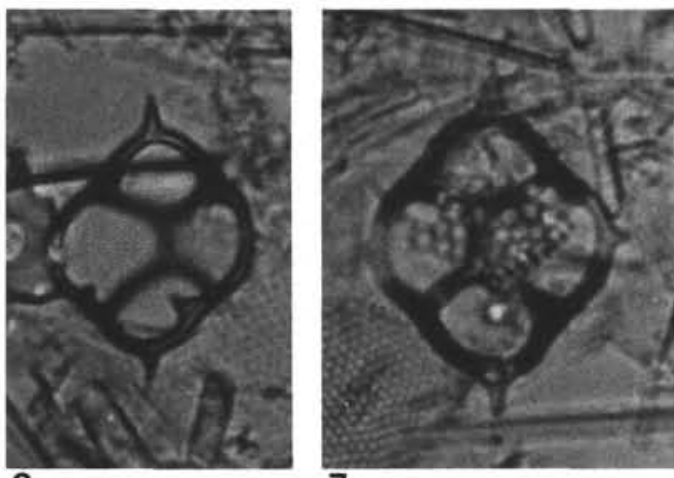

7

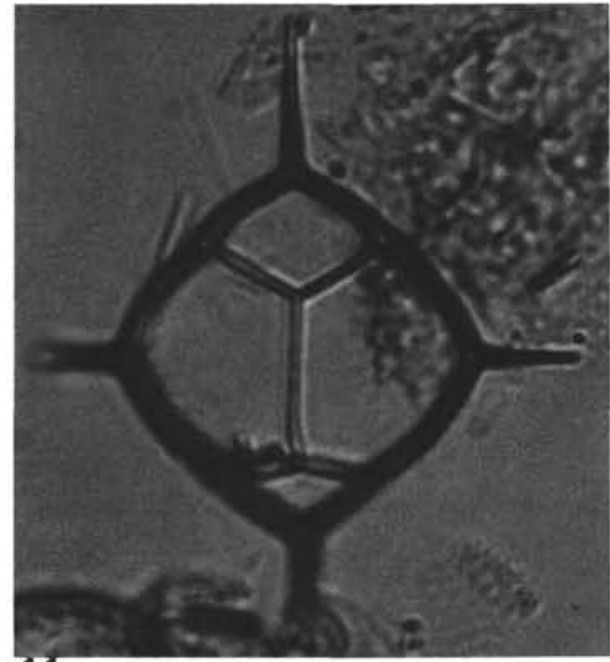

11

3

8
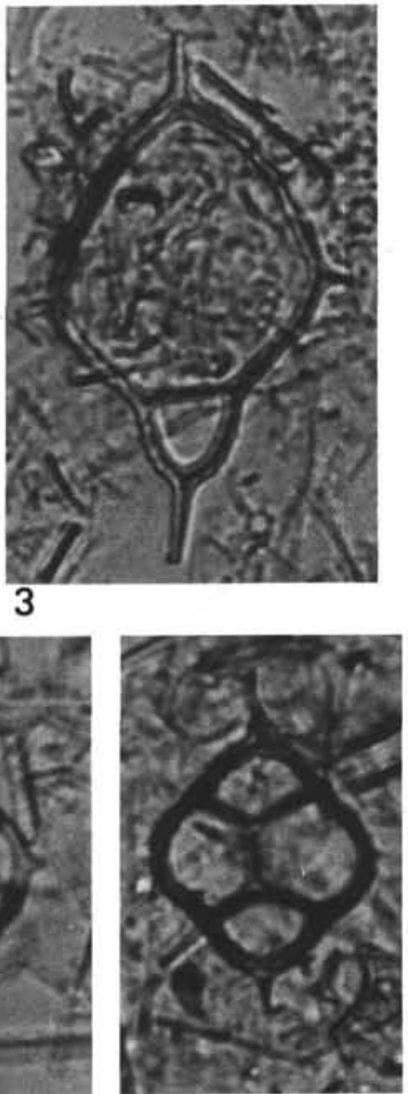

\section{9}

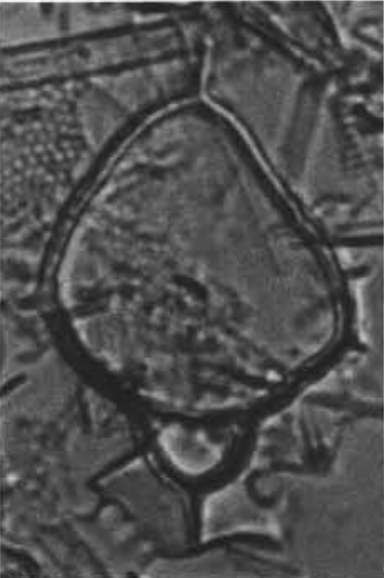

4
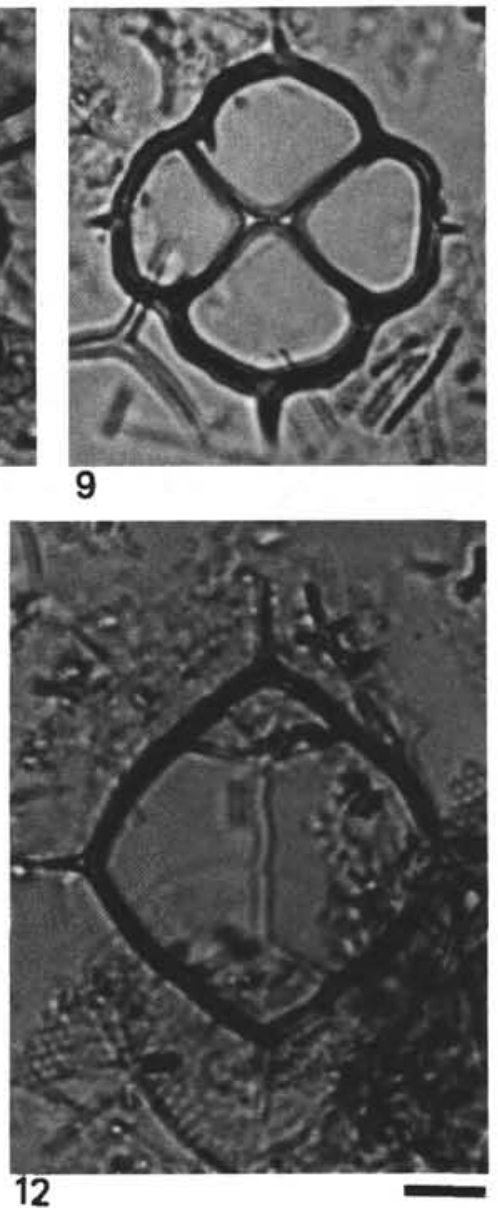

Plate 6. Silicoflagellates from DSDP Hole 504. Scale bar equals $10 \mu \mathrm{m} . \quad 1$. Dictyocha tamarae Bukry, n. sp., USNM 321367, Sample 504-16-2, $42-43 \mathrm{~cm}$. 2. Dictyocha transenna Bukry, Sample 504-48-1, 42-43 cm. 3,4. Dictyocha sp. aff. D. transenna Bukry (spined). (3) Sample 504$52-1,42-43 \mathrm{~cm}$. (4) Sample 504-53-1, 26-27 cm. 5-8. Dictyocha sp. A; consistent small specimens of the Dictyocha spp. category from Sample 504-44-1, 42-43 cm. 9. Dictyocha sp., reminiscent of smaller D. angulata, Sample 504-41-1, 42-43 cm. 10. Dictyocha sp., freak with the portal characteristics of several taxa, such as D. aegea, D. concavata, and D. perlaevis flexatella, Sample 504-50-1, 42-43 cm. 11,12 . Dictyocha spp., possibly mesocenoid transitions from $D$. delicata var. bisecta, Sample 504-33-1, $42-43 \mathrm{~cm}$. 


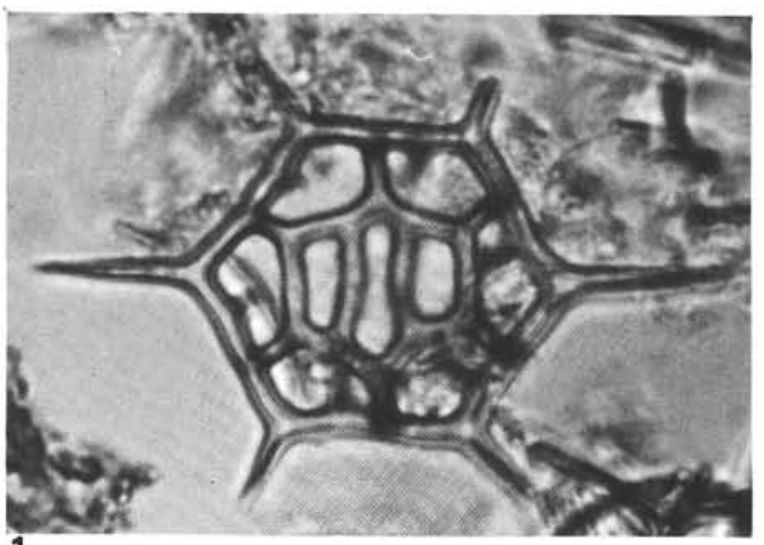

1

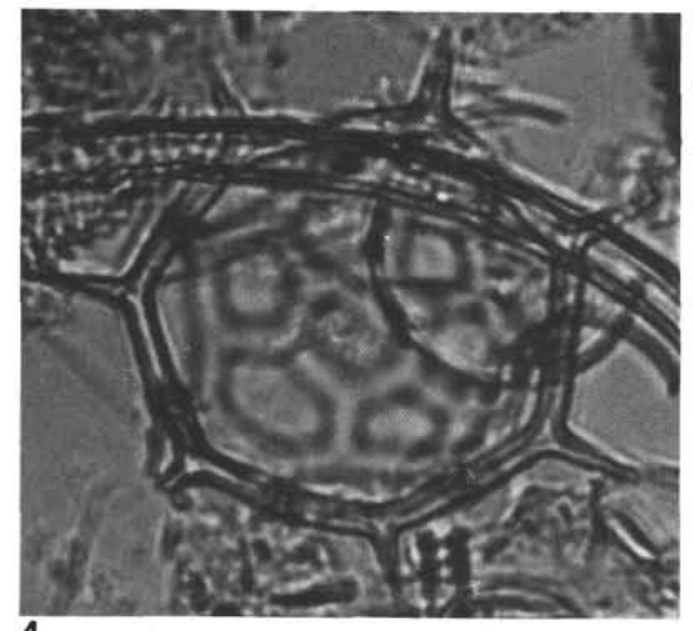

4

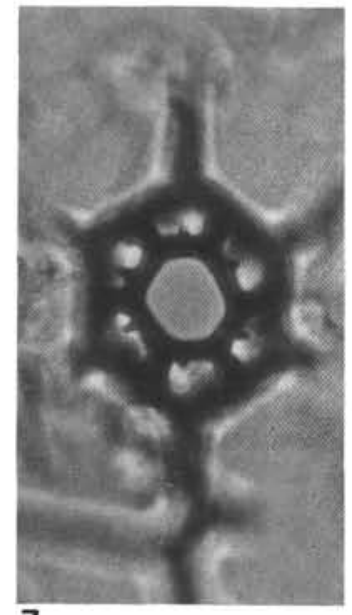
7

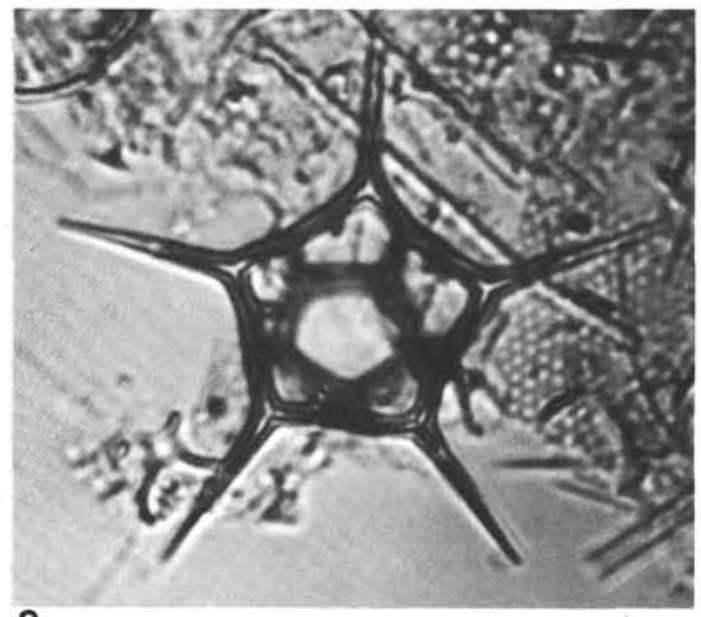

8

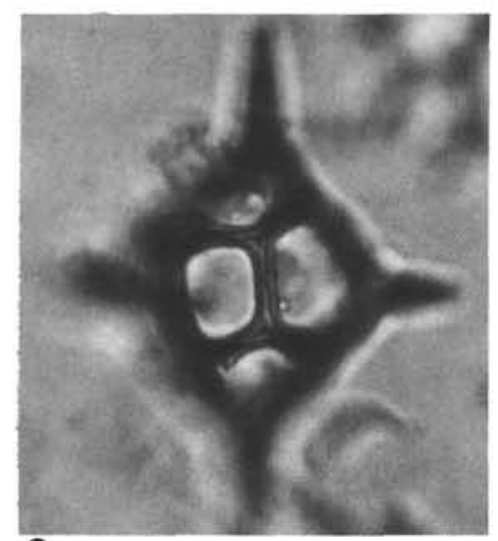

2

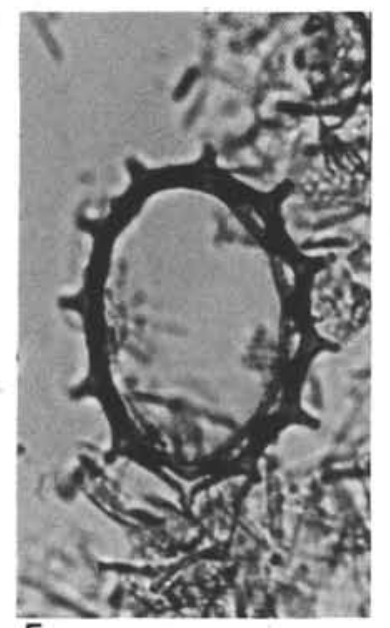

5

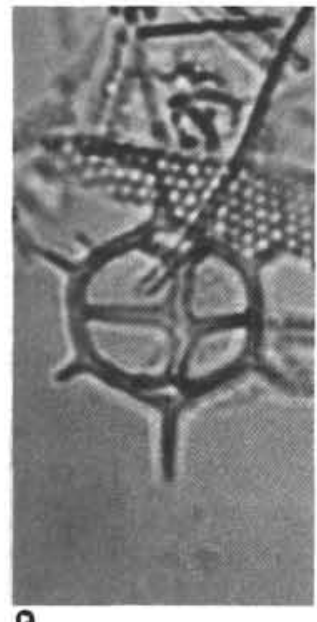

9

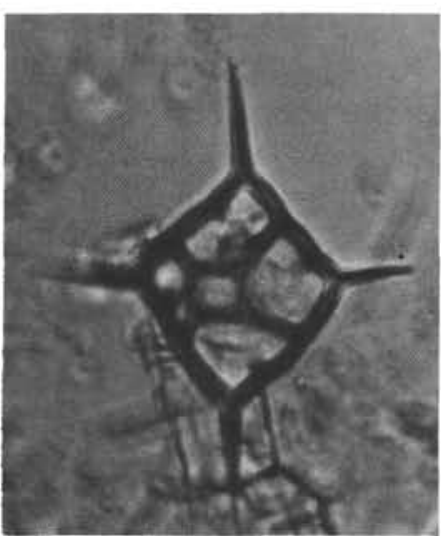

3

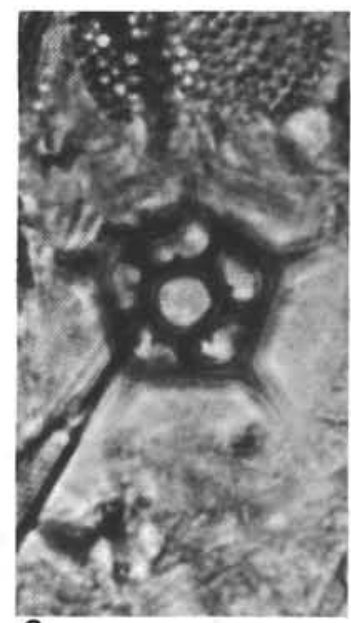

6

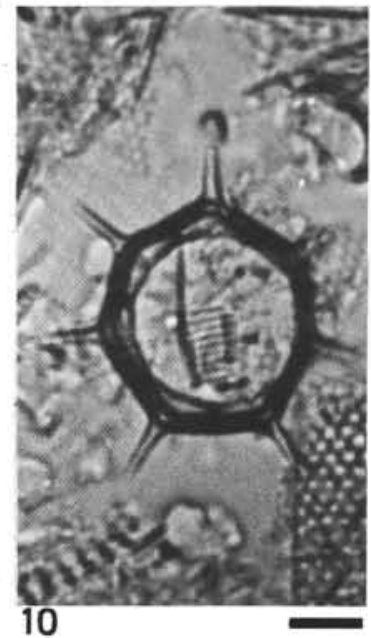

Plate 7. Silicoflagellates from DSDP Hole 504. The same scale bar equals $20 \mu \mathrm{m}$ for Figure 5 and $10 \mu \mathrm{m}$ for Figures 1 through 5 and 7 through 11. 1. Distephanus boliviensis (Frenguelli) (divided ring), Sample 504-10-2, 42-43 cm. 2. Distephanus crux bispinosus Dumitrică, Sample 504-36,CC. 3. Distephanus crux carolae Bukry, Sample 504-42-1, 42-43 cm. 4. Distephanus major (Frenguelli), Sample 504-29-3, 42-43 $\mathrm{cm}$. 5. Distephanus polyactis (Ehrenberg), Sample 504-53-1, 26-27 cm. 6. Distephanus quinquangellus Bukry and Foster, Sample 504-20-2, $42-43 \mathrm{~cm}$. 7. Distephanus speculum f. coronata Schulz, Sample 504-37-1, 42-43 cm. 8. Distephanus sp. A of Bukry (1979a), Sample 504-19-2, 42-43 cm. 9. Distephanus sp. with a bar resembling D. japonica or D. pseudofibula; unique specimen at DSDP Hole 504 observed after counts, Sample 504-40-2, 42-43 cm. 10. Octactis pulchra Schiller, robust basal ring, Sample 504-12-1, 42-43 cm. 


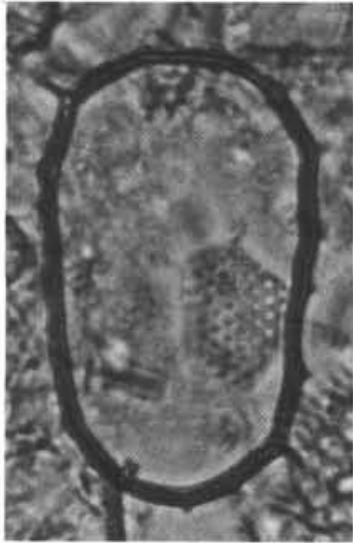
1

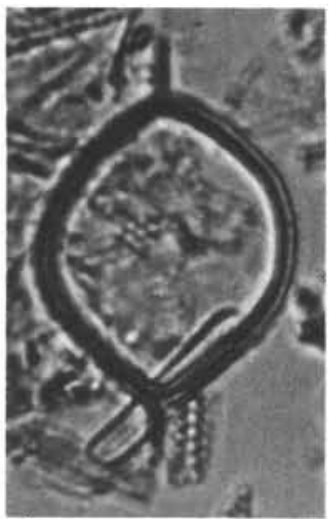

4
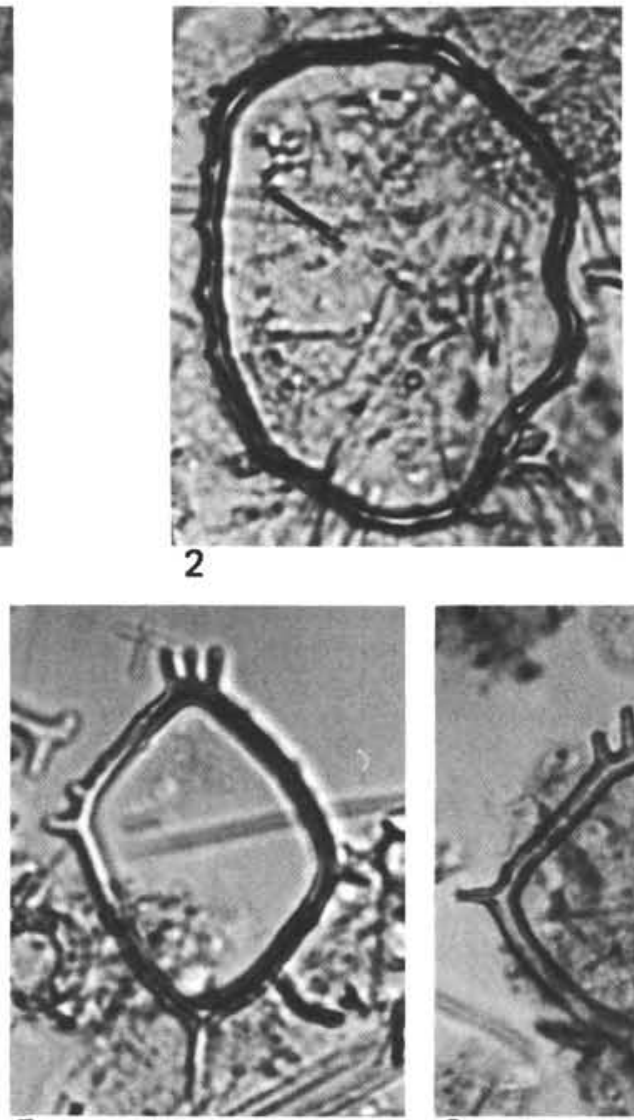

5

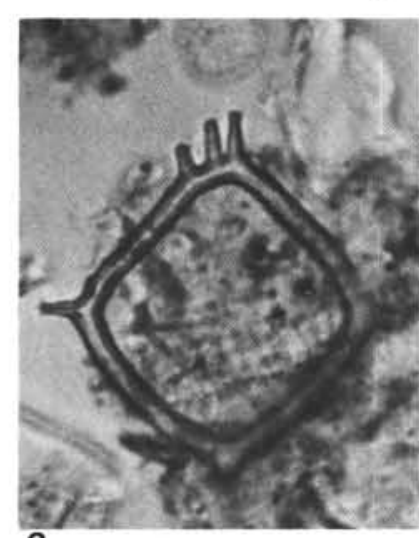

6

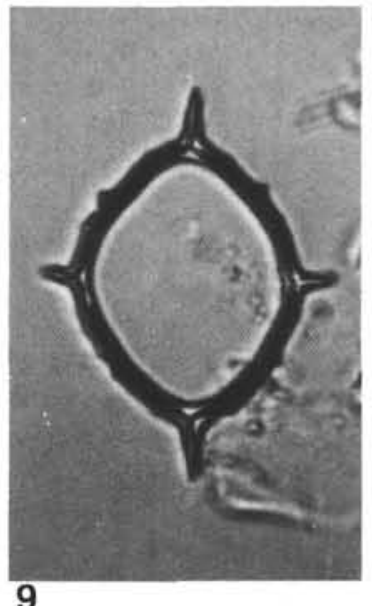

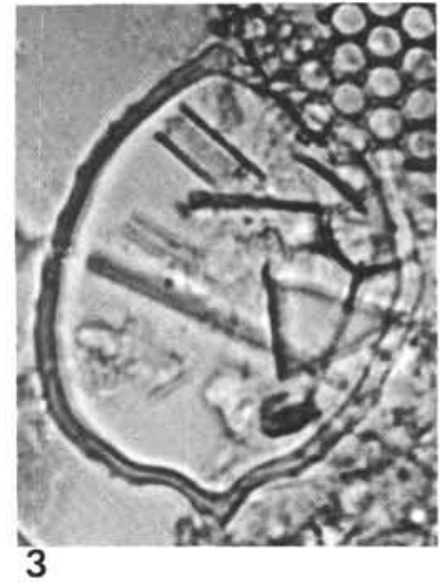

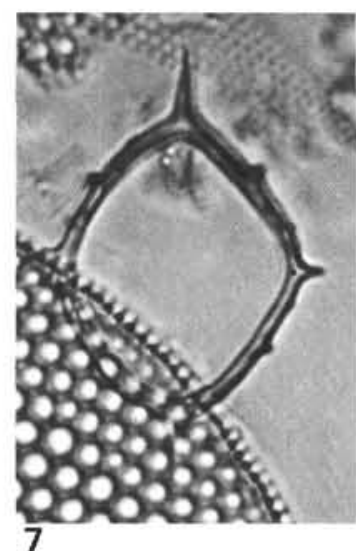

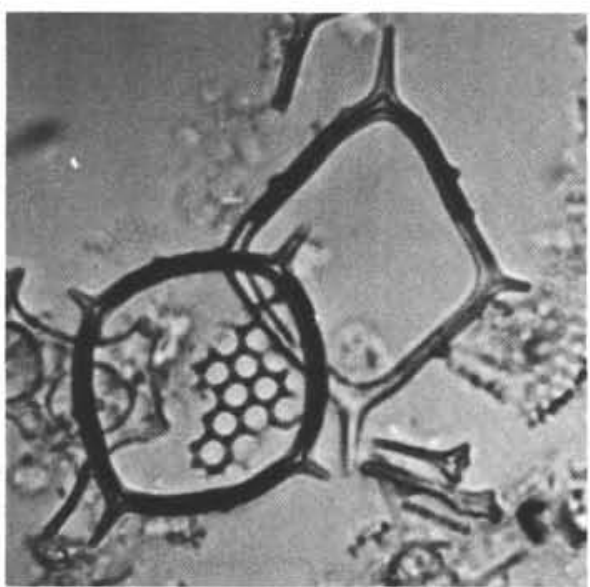

8

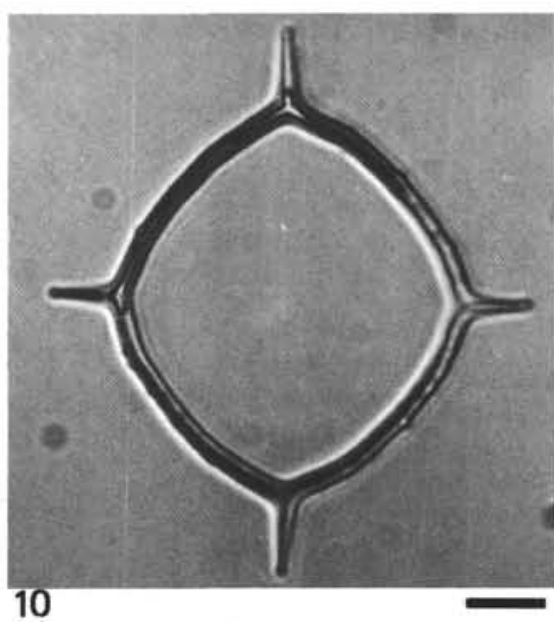

Plate 8. Silicoflagellates from DSDP Hole 504. Scale bar equals $10 \mu \mathrm{m} . \quad 1,2$. Mesocena circulus (Ehrenberg). (1) Oblong with two spines at diagonal corners, Sample 504-43-3, 42-43 cm. (2) Irregular, Sample 504-24-1, $42-43 \mathrm{~cm}$. 3. Mesocena sp. cf. M. circulus (Ehrenberg), Sample 504-5-2, 42-43 cm. 4. Mesocena diodon nodosa Bukry, Sample 504-52-1, 42-43 cm. 5-10. Mesocena quadrangula Ehrenberg ex Haeckel. (5) Multispined, Sample 504-9-2, 42-43 cm. (6) Multispined, Sample 504-10-2, 42-43 cm. (7-9) Small form with four peripheral pikes, all from Sample 504-8-2, $42-43 \mathrm{~cm}$. (10) Typically sized and ornamented form, Sample 504-9-2, $42-43 \mathrm{~cm}$. 

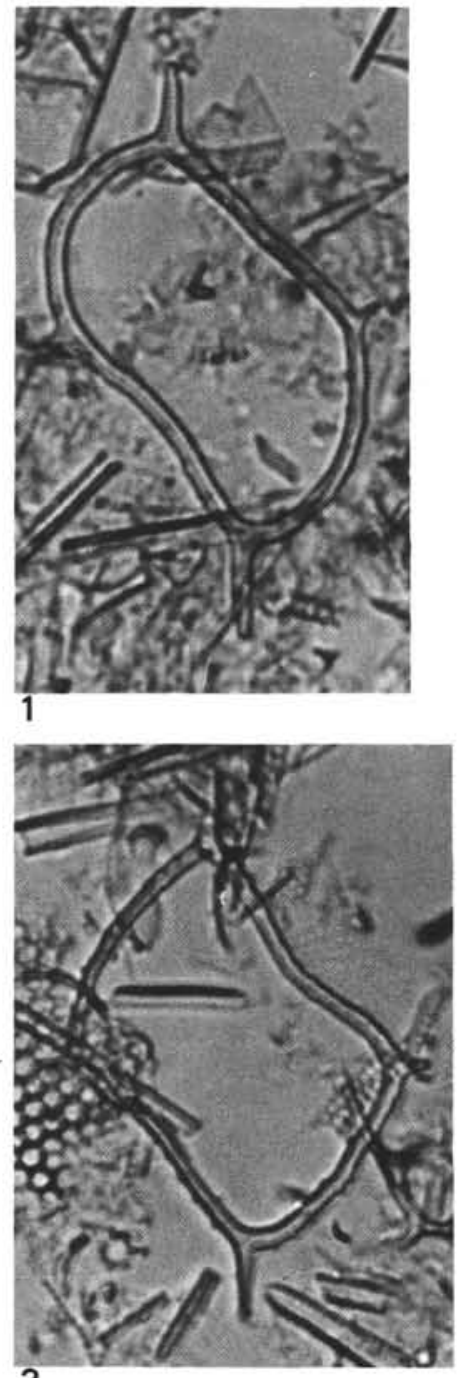

3

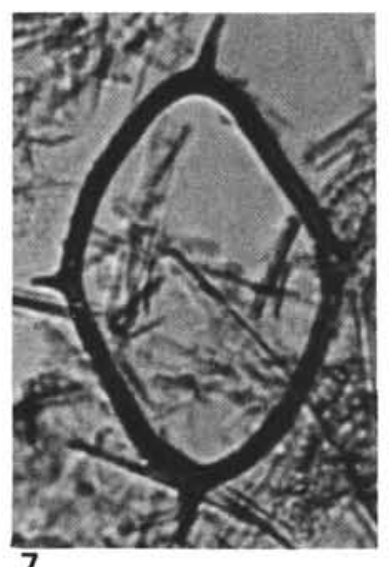

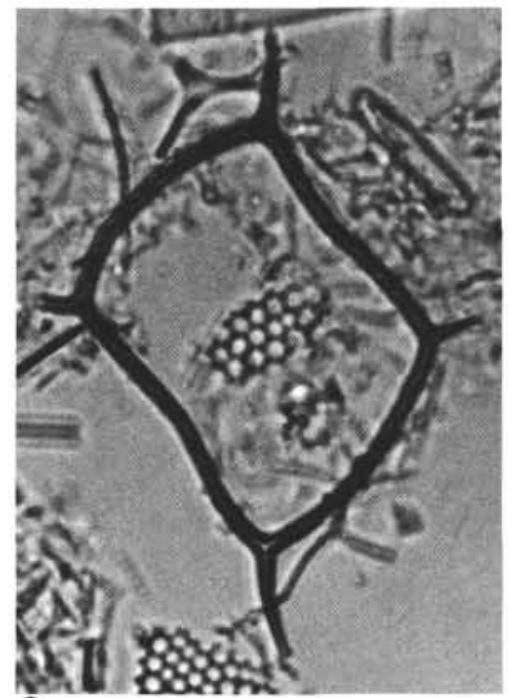

2

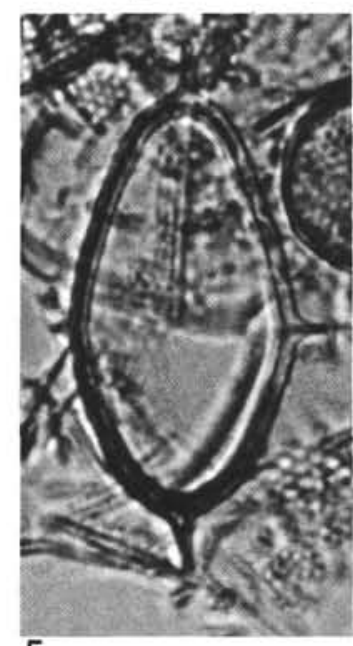

5

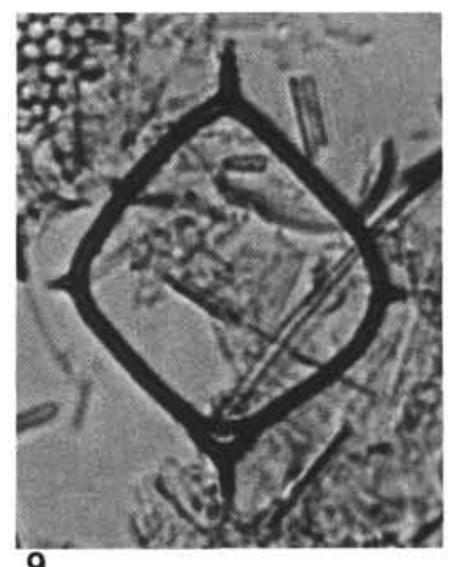

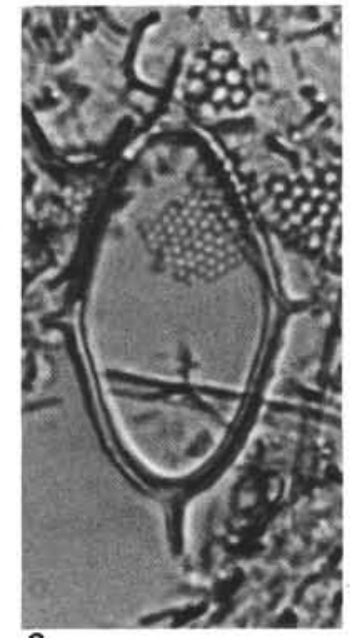

6

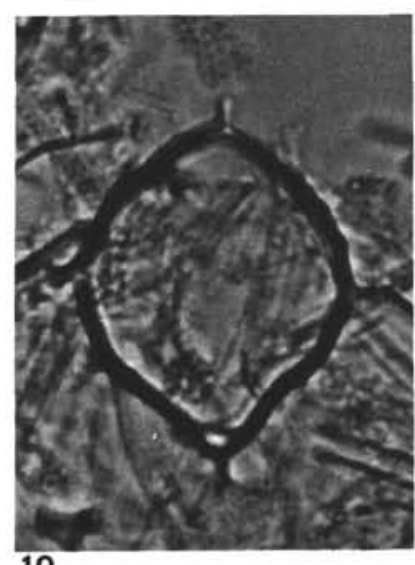

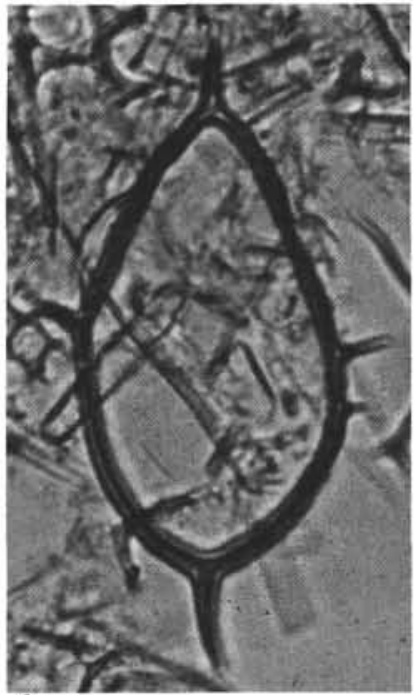

4

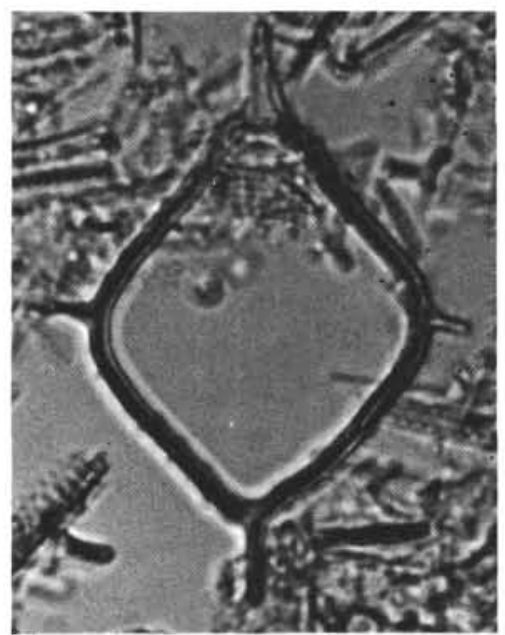

8

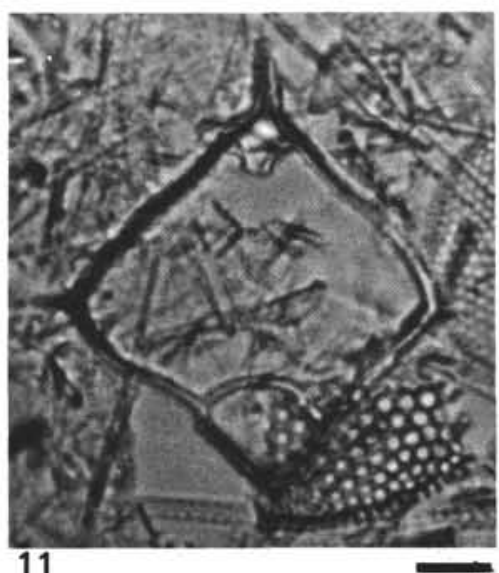

Plate 9. Silicoflagellates from DSDP Hole 504. Scale bar equals $10 \mu \mathrm{m}$. 1-3. Mesocena quadrangula Ehrenberg ex Haeckel (distorted: bowed), Sample 504-52-1, 42-43 cm. 4-7. Mesocena quadrangula Ehrenberg ex Haeckel (distorted: elongate). (4,6) Sample 504-52-1, 42-43 cm. (5,7) Sample 504-53-1, 26-27 cm. 8. Mesocena quadrangula Ehrenberg ex Haeckel, Sample 504-52-1, 42-43 cm. 9-11. Mesocena quadrangula Ehrenberg ex Haeckel (transennoid), possibly derived from Dictyocha. (9) Sample 504-53-1, 26-27 cm. (10) Sample 504-45-1, 42-43 cm. (11) Sample 504-50-1, 42-43 cm. 